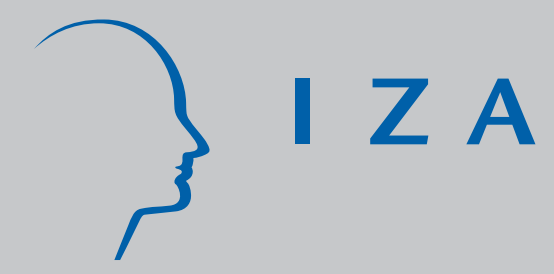

IZA DP No. 1985

Beschäftigung Älterer in Deutschland:

Der unvollständige Paradigmenwechsel

Werner Eichhorst

February 2006 


\title{
Beschäftigung Älterer in Deutschland: Der unvollständige Paradigmenwechsel
}

\section{Werner Eichhorst}

\author{
IZA Bonn
}

\author{
Discussion Paper No. 1985 \\ February 2006
}

\author{
IZA \\ P.O. Box 7240 \\ 53072 Bonn \\ Germany \\ Phone: +49-228-3894-0 \\ Fax: +49-228-3894-180 \\ Email: iza@iza.org
}

\begin{abstract}
Any opinions expressed here are those of the author(s) and not those of the institute. Research disseminated by IZA may include views on policy, but the institute itself takes no institutional policy positions.

The Institute for the Study of Labor (IZA) in Bonn is a local and virtual international research center and a place of communication between science, politics and business. IZA is an independent nonprofit company supported by Deutsche Post World Net. The center is associated with the University of Bonn and offers a stimulating research environment through its research networks, research support, and visitors and doctoral programs. IZA engages in (i) original and internationally competitive research in all fields of labor economics, (ii) development of policy concepts, and (iii) dissemination of research results and concepts to the interested public.
\end{abstract}

IZA Discussion Papers often represent preliminary work and are circulated to encourage discussion. Citation of such a paper should account for its provisional character. A revised version may be available directly from the author. 


\section{ABSTRACT}

\section{Beschäftigung Älterer in Deutschland: Der unvollständige Paradigmenwechsel}

Die Arbeitsmarktintegration älterer Erwerbspersonen ist in Deutschland nach wie vor unbefriedigend. Die vorliegende Analyse erklärt diesen Befund mit institutionellen Einflussgrößen wie Arbeitsmarktregulierung, Transferleistungen, aktiver Arbeitsmarktpolitik sowie Entlohnungsstrukturen und Weiterbildung zu erklären. Es wird prüft, inwieweit sich die institutionellen Rahmenbedingungen für die Beschäftigung Älterer im Zuge der jüngsten Reformen verbessert haben. Handlungsbedarf besteht weiterhin in den Transfersystemen, in der Regulierung des Arbeitsmarktes, der Arbeitsmarktpolitik und im Bereich der arbeitsmarktbezogenen Weiterbildung. Essentiell ist eine konsistente und "altersneutrale“ Rahmensetzung, die den Akteuren auf dem Arbeitsmarkt eindeutige Signale setzt, damit sie sich entsprechend anpassen können.

\section{Employment of Older Workers in Germany: An Incomplete Paradigm Shift}

Despite a sequence of labor market reforms in recent years, employment of older workers in Germany is still lower than in many other European countries. The paper explains this by institutional factors that affect labor supply, labor demand and matching, i.e. labor market regulation, human capital formation, wages as well as the availability of active labor market policy and social benefits. It can be shown that the institutional framework currently in place in Germany is inconsistent. Further reforms are needed to create better employment opportunities for older workers. This implies the abolishment of still existing incentives for early retirement, a more flexible labor law, stronger capacities for human capital formation through "life-long learning" and a consistent strategy to activate older job seekers.

JEL Classification: J26, J48, J68

Keywords: $\quad$ older workers, early retirement, labor market policies, Germany

Corresponding author:

Werner Eichhorst

IZA

P.O. Box 7240

D-53072 Bonn

Germany

Email: eichhorst@iza.org 


\section{Einleitung}

Die Situation älterer Erwerbspersonen auf dem deutschen Arbeitsmarkt ist in den letzten Jahren in den Fokus der wissenschaftlichen und politischen Diskussion gerückt. Auch sind in Deutschland eine Reihe von Veränderungen an den institutionellen Rahmenbedingungen für die Beschäftigung Älterer vorgenommen worden - gleichwohl ist die Entwicklung nach wie vor unbefriedigend. Dieser Beitrag greift auf einen Erklärungsansatz zurück, der institutionelle Einflussgrößen der Beschäftigung Älterer wie Arbeitsmarktregulierung, Transferleistungen, aktive Arbeitsmarktpolitik sowie Entlohnung und Humankapitalinvestitionen in den Mittelpunkt stellt, und prüft, inwieweit sich die institutionellen Rahmenbedingungen für die Beschäftigung Älterer tatsächlich verbessert haben. Dabei wird vor allem auf die Effekte der jüngsten Reformen eingegangen. Abschließend wird der gegenwärtig bestehende Handlungsbedarf aufgezeigt. Es wird deutlich, dass der „Paradigmenwechsel“ zugunsten einer stärkeren Erwerbsintegration Älterer noch längst nicht vollständig realisiert worden ist.

\section{Die Beschäftigungssituation Älterer in Deutschland}

Ältere Erwerbspersonen im Alter von 55 bis 64 Jahren sind in Deutschland deutlich schlechter in den Arbeitsmarkt integriert als jüngere Altersgruppen. Sie treten seltener auf dem Arbeitsmarkt als Anbieter von Arbeit auf und weisen ein niedrigeres Beschäftigungsniveau auf. Hingegen fällt die Arbeitslosigkeit Älterer trotz verschiedener Optionen zum vorzeitigen Ausstieg aus dem Erwerbsleben etwas höher aus (Abbildung 1).

- Abbildung 1 hier einfügen -

Der Befund eines massiven Beschäftigungsdefizits gilt nach wie vor, obwohl in den letzten Jahren ein leichter Anstieg der Erwerbstätigkeit Älterer zu beobachten war. Dieser Zuwachs geht einerseits darauf zurück, dass stärker in den Arbeitsmarkt integrierte Kohorten in die Altersgruppe der 55- bis 64-Jährigen hineingewachsen sind; andererseits nimmt die Erwerbstätigkeit älterer Frauen in Teilzeitarbeit und geringfügiger Beschäftigung (Minijobs) zu (Büttner 2005a, 2005b). Unverändert kann jedoch von einem deutlichen Abstand 
des Beschäftigungsniveaus der Älteren zur jüngeren und mittleren Altersgruppe gesprochen werden. Dies gilt insbesondere für Männer und gering qualifizierte Ältere, wie Tabelle 1 belegt (OECD 2005).

- Tabelle 1 hier einfügen -

Insgesamt resultiert eine im internationalen Vergleich unterhalb des Durchschnitts und der EU-Zielgröße von 50\% liegende und nur mäßig steigende Beschäftigungsquote aller Personen im Alter von 55 bis 64 Jahren (Abbildung 2).

- Abbildung 2 hier einfügen -

Obwohl das Risiko, arbeitslos zu werden, bei Älteren geringer ist, fällt die durchschnittliche Dauer der Arbeitslosigkeit wesentlich länger aus als bei jüngeren Erwerbspersonen (Brussig/Knuth/Schweer 2006). Dies schlägt sich in hoher Langzeitarbeitslosigkeit nieder (Abbildung 3), welche jedoch zum Teil über Transferleistungen abgesicherte Frühverrentung darstellt (OECD 2005).

- Abbildung 3 hier einfügen -

Im internationalen Vergleich ergibt sich so ein mittleres, jedoch steigendes Erwerbsaustrittsalter von zuletzt 61,3 Jahren. Es gibt an, zu welchem Zeitpunkt Erwerbstätige aus ihrer letzten Beschäftigung ausscheiden (Abbildung 4). Der Anstieg des Erwerbsaustrittsalters blieb damit hinter dem Anstieg der Lebenserwartung zurück; die Differenz zur Regelaltersgrenze von 65 Jahren ist mit knapp vier Jahren noch immer erheblich.

\section{- Abbildung 4 hier einfügen -}

Ältere sind also in Deutschland sowohl im Vergleich zu anderen Altersgruppen als auch zu älteren Erwerbspersonen in vielen anderen Ländern schlechter in den Arbeitsmarkt integriert. Gleichwohl befindet sich Deutschland in einer Übergangssituation. Ein Teil des Rückstands bei der Beschäftigungsquote und dem Erwerbsaustrittsalter konnte bereits aufgeholt werden. Die Anstiege bei geringfügiger und Teilzeitarbeit deuten jedoch an, dass 
bei vollwertiger Erwerbsintegration bislang wenig erreicht wurde. Über Transferleistungen abgesicherte Nichterwerbstätigkeit und Arbeitslosigkeit spielt als Übergangsphase in den Ruhestand noch immer eine bedeutende Rolle. Die Phase bis zum gesetzlichen Rentenalter bzw. zum vorgezogenen Renteneintritt wird durch Transferleistungen aus der Arbeitslosenversicherung überbrückt, teilweise ohne dass dies in der Arbeitslosenstatistik ausgewiesen wird. Die Wiederbeschäftigungschancen älterer Arbeitsloser sind entsprechend begrenzt. Damit gehen ein Verlust an produktiv einsetzbarem Erwerbspersonenpotenzial und eine erhebliche Belastung der Sozialsysteme einher (Gruber/Wise 2005). Angesichts des demographischen Wandels, der in einer Alterung und Schrumpfung der Bevölkerung im erwerbsfähigen Alter resultieren wird (OECD 2005), muss eine bessere Arbeitsmarktintegration auch im späteren Verlauf des Erwerbslebens ein wichtiges Ziel der Beschäftigungspolitik sein.

\section{Ein institutionalistischer Erklärungsansatz}

Die Unterschiede im Niveau und der Entwicklung der Erwerbstätigkeit älterer Erwerbspersonen im Vergleich zu jüngeren Erwerbspersonen, aber auch im internationalen Vergleich, lassen sich gut über institutionelle Einflussfaktoren erklären (Funk 2004, Gruber/Wise 1999, Ebbinghaus 2002, Arnds/Bonin 2003a). Institutionelle Rahmenbedingungen beeinflussen die Entscheidung zur Weiter- oder Wiederbeschäftigung in der späten Phase des Erwerbslebens, indem sie Kosten und Nutzen zusätzlicher Beschäftigung auf Seiten der Arbeitgeber und der Beschäftigten determinieren. Die Entscheidung über Arbeitsnachfrage und Arbeitsangebot lässt sich im Kern auf das ökonomische Kalkül von Arbeitgebern und Arbeitnehmern zurückführen.

Aus Sicht der Arbeitgeber wird ein älterer Arbeitnehmer dann eingestellt oder weiterbeschäftigt, wenn die Arbeitskosten der Weiterbeschäftigung bzw. Neueinstellung nicht höher liegen als die Arbeitsproduktivität. Auf die Arbeitskosten wirken Bruttolöhne und Lohnnebenkosten sowie Einstellungs- und Entlassungskosten ein. Die Arbeitsproduktivität hängt vom individuellen Humankapital und der Arbeitsorganisation ab, d.h. vom betriebliche Personaleinsatz. Es ist davon auszugehen, dass sowohl bei der Beurteilung der Arbeitskosten als auch bei der Einschätzung der Arbeitsproduktivität von Arbeitnehmern auf Seiten der (potenziellen) Arbeitgeber neben objektiven Informationen auch subjektive Einschätzungen einfließen. Was die Arbeitskosten angeht, so kann ein starker Anstieg der Ent- 
lohnung nach Betriebszugehörigkeit oder Lebensalter, etwa auf tariflicher Basis, die Arbeitnachfrage dämpfen, wenn dies nicht durch eine entsprechende Arbeitsproduktivität auf der Grundlage betrieblich nutzbaren Humankapitals gerechtfertigt ist. Senioritätslöhne sind jedoch betriebswirtschaftlich plausibel, wenn damit eine Bindung der Arbeitnehmer an den Betrieb erreicht werden kann oder die Produktivität nach längerer Betriebszugehörigkeit ein höheres Niveau erreicht.

Die Regulierung des Arbeitsmarktes in Gestalt des Kündigungsschutzes kann in zwei Richtungen wirken: Einerseits können tatsächlich oder vermeintlich höhere Entlassungskosten die Weiterbeschäftigung von eingearbeiteten Arbeitskräften begünstigen; andererseits kann ein strikter Kündigungsschutz Neueinstellungen hemmen, da Arbeitskräfte weniger leicht entlassen werden können. Sind befristete Beschäftigungsverhältnisse möglich, so dürfte dies die Einstellungsschwelle vermindern.

Die Produktivität wiederum hängt zunächst von der Qualifikation der Arbeitskräfte ab, die durch Erstausbildung und Weiterbildung bestimmt wird. Je mehr die Qualifikation den aktuellen Anforderungen des Betriebs entspricht, um so eher werden Arbeitskräfte weiter beschäftigt oder neu eingestellt. Wird die Qualifikation hingegen im Strukturwandel entwertet, ohne dass dies durch eine Anpassung des Humankapitals kompensiert wird, so sinken die Chancen auf Weiter- oder Wiederbeschäftigung. Investitionen in das Humankapital durch Arbeitgeber und Arbeitnehmer fallen umso geringer aus, je mehr die Verfügbarkeit von Transferleistungen einen frühzeitigen Ausstieg aus dem Erwerbsleben ermöglicht.

Die Entscheidung zur Weiterarbeit bzw. zur Wiederaufnahme einer Beschäftigung hängt aus Sicht der Erwerbspersonen vor allem vom erzielbaren Arbeitsverdienst im Vergleich zu den verfügbaren Einkommensersatzleistungen aus den sozialen Sicherungssystemen ab. Je attraktiver die Transferleistungen und je weniger eine Verlängerung des Erwerbslebens zu höheren Transferansprüchen zu einem späteren Zeitpunkt beiträgt, umso eher wird eine Erwerbsperson aus dem Arbeitsmarkt ausscheiden und umso weniger wird sie an der Aufnahme einer neuen Beschäftigung interessiert sein. Dabei beeinflusst ein möglicher Anstieg der Entlohnung mit dem Lebensalter oder der Betriebszugehörigkeit im Zusammenwirken mit dem Transfersystem die Höhe der Anspruchslöhne, d.h. des aus Sicht des Arbeitnehmers akzeptablen Lohnes. Die Entlohnung aus fortgesetzter Erwerbstätigkeit konkurriert damit aus Sicht des Arbeitnehmers mit der Verfügbarkeit und der Großzügigkeit von Transferleistungen der Arbeitslosen- oder Rentenversicherung. Wird der Zugang zu den Einkommensersatzleistungen im Zuge einer „aktivierenden“ Arbeitsmarktpolitik erschwert oder werden die Transfers in ihrer Höhe beschnitten, so sinken tendenziell die An- 
spruchslöhne. Ist der vorzeitige Renteneintritt mit erheblichen Abschlägen, ein späteres Ausscheiden aber mit höheren Renten verbunden, so stellt dies einen Anreiz zu längerer Erwerbstätigkeit dar (Gruber/Wise 1999, 2005, Duval 2003).

Arbeitsnachfrage und Arbeitsangebot werden auf dem Arbeitsmarkt über Matching ausgeglichen. Dabei kommen der Lohnbildung und der Arbeitsmarktpolitik zentrale Funktionen zu. Die Entlohnung muss gleichzeitig der Produktivität entsprechen und dem Vergleich mit alternativ verfügbaren Transferleistungen standhalten. Die aktive Arbeitsmarktpolitik kann die Wiederbeschäftigungschancen Älterer verbessern, indem sie Qualifikationsdefizite durch Weiterbildungsmaßnahmen ausgleicht, Kosten- oder Produktivitätsnachteile durch Lohnkostenzuschüsse kompensiert oder niedriger entlohnte Beschäftigung durch aufstockende Transfers attraktiver macht. Daneben kann sie über die Unterstützung und Kontrolle der Arbeitssuche sowie Stellenvermittlung zur Arbeitsaufnahme beitragen. Dieser Erklärungsrahmen beschränkt sich bewusst auf institutionelle Faktoren und geht nicht auf andere Motive für die Einstellung oder Entlassung von Personal bzw. die Aufnahme einer Erwerbsarbeit ein. Er lässt jedoch zu, dass es zur „Diskriminierung“ Älterer durch bestimmte institutionelle Regelungen kommen kann, welche in der Wahrnehmung der Arbeitgeber die Neigung zur Weiter- oder Wiederbeschäftigung Älterer vermindern.

\section{Rahmenbedingungen der Beschäftigung Älterer in Deutschland}

In Deutschland sind bei zentralen institutionellen Rahmenbedingungen für die Beschäftigung Älterer während der letzten Jahre eine Reihe von Reformen verabschiedet und umgesetzt worden. Gleichwohl bleibt zur prüfen, inwieweit diese Reformen zu einer Verbesserung der Arbeitsmarktchancen Älterer beigetragen haben.

\subsection{Regulierung des Arbeitsmarktes}

Die Regulierung des Arbeitsmarktes betrifft zunächst den individuellen Kündigungsschutz. Nach dem deutschen Kündigungsschutzgesetz gelten bei langer Betriebszugehörigkeit längere Kündigungsfristen. Lange Betriebszugehörigkeit und höheres Lebensalter werden neben Unterhaltspflichten und Schwerbehinderung auch als Kriterien der Sozialauswahl herangezogen. Wenn bei den vom Kündigungsschutz erfassten Beschäftigten davon auszu-

gehen ist, dass ein höheres Lebensalter nach wie vor in vielen Fällen mit längerer Betriebs- 
zugehörigkeit einhergeht, bedeutet das einen besonderen Bestandsschutz für Arbeitsverhältnisse älterer Arbeitnehmer. Durch tarifvertragliche Regelungen kann darüber hinaus, etwa im öffentlichen Dienst, nach einer bestimmten Dauer der Betriebszugehörigkeit oder ab einem bestimmten Alter eine ordentliche Kündigung ganz ausgeschlossen sein. Wird der Kündigungsschutz mit Lebensalter und Betriebszugehörigkeit stärker, so wird die Entlassung älterer Arbeitnehmer für den Arbeitgeber schwieriger und teurer. Gleichzeitig kann der Kündigungsschutz jedoch Anreize dafür bieten, durch betriebliche Weiterbildung firmenspezifisches Humankapital zu entwickeln (Estevez-Abe/Soskice/Iversen 2001). Die rechtliche Stabilisierung des Arbeitsverhältnisses und die Humankapitalwirkungen des Kündigungsschutzes können damit die Chance der Weiterbeschäftigung im selben Betrieb erhöhen.

Ist es jedoch schwierig, einen älteren Arbeitnehmer zu kündigen, so greifen Unternehmen vermehrt auf Auflösungsverträge in Verbindung mit Abfindungszahlungen zurück, deren Höhe mit dem Entlohnungsniveau, der Betriebszugehörigkeit und dem Alter zunimmt (Jahn 2005). Sie waren bis Ende 2005 innerhalb bestimmter Höchstgrenzen steuerlich begünstigt; auch kann durch eine geeignete Gestaltung die Zahlung von Beiträgen zur Sozialversicherung und eine Anrechnung der Abfindungen auf das Arbeitslosengeld verhindert werden (§§ 143 a und 144 SGB III). In Verbindung mit staatlichen Transferleistungen kann dies unter Vermeidung einer Kündigung zum Abbau älterer Arbeitskräfte auf Kosten der Sozialsysteme führen, solange auf eine längere Bezugsdauer von Arbeitslosengeld I ohne Verfügbarkeit für den Arbeitsmarkt (§ 428 SGB III) und anschließend eine vorgezogene Rente wegen Arbeitslosigkeit zurückgegriffen werden kann. Auf der anderen Seite ist anzunehmen, dass die Bereitschaft der Arbeitgeber, ältere Arbeitnehmer neu einzustellen geringer ausfällt, wenn - gegeben das hohe Maß an Rechts- und Kostenunsicherheit im deutschen Kündigungsschutzrecht - Ältere tendenziell schwerer entlassen werden können. Der Schutz älterer Arbeitnehmer durch den Kündigungsschutz kann sich aufgrund dieser Zusammenhänge und deren Wahrnehmung durch potenzielle Arbeitgeber gegen die Wiederbeschäftigungschancen dieser Gruppe nach einem Arbeitsplatzverlust richten. International vergleichende Studien sprechen für diesen Zusammenhang (OECD 2004). Die Wirkungen des Kündigungsschutzes sind negativer einzuschätzen, wenn das ökonomische Umfeld turbulenter wird, sich Betriebsschließungen und Entlassungen weniger vermeiden lassen und deshalb auch ältere Arbeitskräfte auf eine Gelegenheit zur Wiederbeschäftigung angewiesen sind. 
Allerdings müssen ältere Arbeitnehmer bei einer Einstellung nicht direkt in ein unbefristetes und vom Kündigungsschutz erfasstes Arbeitsverhältnis aufgenommen werden. Um die negativen Wirkungen des Kündigungsschutzes auf die Wiedereinstellung Älterer zu vermindern, wurde im Zuge der Hartz-Reformen 2003 mit § 14 Abs. 3 des Teilzeit- und Befristungsgesetzes bis Ende 2006 die Möglichkeit eröffnet, Arbeitnehmer statt ab 58 Jahren bereits ab 52 Jahren ohne sachlichen Grund befristet beschäftigen zu können, während dies für jüngere Arbeitnehmer nur für maximal zwei Jahre zulässig ist. Unter Einbezug der ohnehin möglichen Befristung von zwei Jahren ohne sachlichen Grund, konnten Arbeitskräfte bereits ab 50 Jahren ohne Beschränkungen mehrfach hintereinander befristet eingestellt werden. Mittlerweile vorliegende Evaluationsergebnisse (RWI/ISG 2005) zeigen auf der Basis eines sehr kurzen Beobachtungszeitraums keine Effekte der neuen Befristungsregelung im Sinne eines höheren Anteils befristet Beschäftigter in dieser Altersgruppe. 95\% der im Rahmen der Evaluation befragten Unternehmen gaben an, dass die Reform ihr Einstellungsverhalten gegenüber älteren Arbeitskräften nicht verändert habe. In der Praxis spielt dieses Instrument aufgrund seiner geringen Bekanntheit ohnehin nur eine sehr marginale Rolle. Die befragten Unternehmen plädierten in diesem Zusammenhang eher für Lockerungen des Kündigungsschutzes und individuelle Lohnanpassungen. Die erleichterte Befristung bei älteren Arbeitnehmern muss nun ohnehin korrigiert werden, da der Europäische Gerichtshof in seinem Urteil vom 22. November 2005 (Az.: C-144/04) festgestellt hat, dass die Förderung der Beschäftigung älterer Arbeitskräfte es nicht rechtfertige, diese vom Kündigungsschutz auszunehmen. Dies stellt in den Augen des EuGH eine unzulässige Diskriminierung aufgrund des Alters dar und ist damit nichtig.

\subsection{Entlohnung}

Verfügbare Informationen zeigen für Deutschland keine ausgeprägte Senioritätsentlohnung, d.h. einen Anstieg des Arbeitsentgelts mit dem Lebensalter. Eine Ausnahme hiervon stellte der öffentliche Dienst nach altem Tarifvertrag dar. Hier hat der neue Tarifvertrag jedoch eine Abschwächung mit sich gebracht. Deshalb kann man nicht von ausgeprägter Senioritätsentlohnung in Deutschland sprechen (OECD 2005). Häufiger sind jedoch tarifvertragliche Regelungen über einen Anstieg der Entlohnung nach der Dauer der Betriebszugehörigkeit, wie dies aus betrieblicher Sicht auch sinnvoll sein kann. Bei „Normalarbeitsverhältnissen“ mit längerer Betriebszugehörigkeit ergibt sich damit ein positiver Zu- 
sammenhang zwischen Entlohnung und Alter. Bei insgesamt hoher Abgabenbelastung kann von den Lohnnebenkosten ein zusätzlicher negativer Effekt auf die Arbeitsnachfrage ausgehen (OECD 2005). Ältere können jedoch bei Neueinstellungen grundsätzlich ohne Rücksicht auf ihr Lebensalter zu niedrigeren Löhnen eingestellt werden als die im jeweiligen Betrieb bereits Beschäftigten gleichen Alters.

Allerdings gibt es in der Praxis dennoch zwei Probleme: die Anspruchslöhne Älterer und die Einordnung in das betriebliche Gehaltsgefüge. Löhne nach Betriebszugehörigkeit können die Aufnahme einer neuen Stelle für Ältere weniger attraktiv machen, denn sie haben tendenziell höhere Anspruchslöhne, weil sie von längerer Betriebszugehörigkeit bei ihrem bisherigen Arbeitgeber profitiert hatten, bevor sie arbeitslos wurden (Brixy/Christensen 2002). Dieser „Sperrklinkeneffekt“ der Senioritätslöhne im früheren Betrieb kann die Aufnahme vergleichsweise geringer entlohnter Arbeit durch Ältere bzw. die Besetzung der ihnen angebotenen Stellen erschweren. Dieser Effekt hängt jedoch auch von der Höhe der verfügbaren Transferleistungen ab. Zusätzlich müssen Arbeitgeber bei Neueinstellungen Älterer eine Einordnung in das betriebliche Entlohnungsgefüge vornehmen, um innerbetriebliche Konflikte zu vermeiden. Ohne dass die Arbeitgeber formal dazu gezwungen wären, kann das zur Gewährung von Arbeitsentgelt führen, das Beschäftigte ähnlichen Alters mit längerer Betriebszugehörigkeit erhalten, aber bei Neueinstellungen mit entsprechendem Einarbeitungsbedarf die anfängliche Produktivität übersteigen kann.

\subsection{Transferleistungen}

Verschiedene Frühverrentungsoptionen, welche in den 1970er und 1980er Jahren eröffnet wurden, sind in den letzten Jahren bereits abgebaut worden (Eichhorst/Sproß 2005, OECD 2005). Dies gilt insbesondere für die Rentenreformen 1992 und 1999, welche zwischen 1997 und 2004 in Kraft traten. Bei vorgezogenem Renteneintritt bedeutete dies versicherungsmathematisch annähernd, jedoch nicht vollkommen korrekte Abschläge (BörschSupan 2004, OECD 2005). Daneben wurden verschiedene Optionen des vorzeitigen Renteneintritts vor dem gesetzlichen Rentenalter von 65 Jahren geschlossen. Das gesetzliche Rentenalter soll nach den jüngsten Beschlüssen für jüngere Jahrgänge bis auf 67 steigen. Die Altersgrenze für den Eintritt in Rente wegen Arbeitslosigkeit wird gegenwärtig bis 2008 von 60 auf 63 Jahre angehoben. 2016 wird diese Rentenform ganz auslaufen. Gleiches gilt für die Rente nach Altersteilzeit und die vorgezogene Altersrente für Frauen. Wei- 
terhin besteht jedoch die Rente für langjährig Versicherte mit mindestens 35 Versicherungsjahren ab 63 Jahren. Ab 2010 soll diese bereits ab 62 Jahren verfügbar sein. Die Rente für Schwerbehinderte ist ab 63 ohne Abschläge, mit Abschlägen ab 60 Jahren zugänglich. Neben der Veränderung der Altersgrenzen und den stärkeren Abschlägen macht auch die allgemein sinkende Höhe der zu erwartenden Renten einen vorzeitigen Ausstieg weniger attraktiv.

Die früheren Erwerbsunfähigkeitsrenten wurden 2001 durch Erwerbsminderungsrenten ersetzt. Dies bedeutete die grundsätzlich nur noch befristete Gewährung dieser Renten und für Jüngere den Wegfall des Berufsschutzes, der jedoch für derzeit 45-Jährige und Ältere immer noch gilt. Bei teilweiser Erwerbsminderung mit einem Leistungsvermögen von drei bis sechs Stunden pro Tag wird auch die Arbeitsmarktlage bei der Entscheidung über die Rentenhöhe mit berücksichtigt. Liegt neben eingeschränkter Leistungsfähigkeit auch Arbeitslosigkeit vor, so wird der Teilzeitarbeitsmarkt vom Bundessozialgericht in ständiger Rechtsprechung (z.B. BSG SozR 3-5868 § 13 Nr. 1 und BSG SozR 3-5750 Art. 2 § 6 Nr. 6, 10) ohne weiteren Nachweis der Stellensuche oder -vermittlung als verschlossen angesehen. Unter der regelmäßigen Annahme eines verschlossenen Teilzeitarbeitsmarktes für diesen Personenkreis wird bei teilweise Erwerbsgeminderten über 60 Jahren grundsätzlich eine volle Erwerbsminderungsrente gewährt.

Weiterhin möglich ist auch das vorgezogene Ausscheiden über die Altersteilzeit ab 55 Jahren bis zum Renteneintritt, welche aus Mitteln der Arbeitsmarktpolitik für maximal sechs Jahre gefördert werden kann. Während der Phase der Altersteilzeit werden das verminderte Arbeitsentgelt um mindestens 20\% und die Rentenbeiträge auf 90\% des vorigen Entgelts vom Arbeitgeber aufgestockt. Er bekommt dies von der Bundesagentur erstattet, wenn er einen Arbeitslosen einstellt oder einen Auszubildenden übernimmt. Dies ist nach gegenwärtigem Stand noch bis Ende 2009 möglich. In der Praxis führt die Altersteilzeit in ihrer weit überwiegenden Nutzung als „Blockmodell“ nicht zu einem graduellen Übergang, sondern zu einem vorgezogenen Ausstieg aus dem Erwerbsleben zu Beginn der Freistellungsphase. Die erwarteten Beschäftigungseffekte zugunsten jüngerer Arbeitnehmer sind fraglich. Allerdings wird nur in einem Drittel bis zur Hälfte der Fälle eine Förderung durch die BA beansprucht.

Die Bezugsdauer des Arbeitslosengeldes I ist mit Wirkung vom Februar 2006 nach einer Übergangsphase von 32 auf maximal 18 Monate für Arbeitslose über 55 Jahren verkürzt worden, was im Gegensatz zur Ausdehnung in den 1980er Jahren lange Arbeitslosigkeit weniger attraktiv macht. Danach greift das Arbeitslosengeld II mit strikten Zumutbarkeits- 
kriterien, die jedoch bei Älteren bislang kaum angewandt wurden. Allerdings wurde Ende 2005 auch die so genannte „58er-Regelung“ des § 428 SGB III um weitere zwei Jahre bis Ende 2007 verlängert. Sie gestattet den Bezug von Arbeitslosengeld I oder II ohne für den Arbeitsmarkt verfügbar sein zu müssen, sofern zum frühestmöglichen Zeitpunkt eine ungeminderte Altersrente beantragt wird. Bereits Anfang 2004 führte der Übergang vom Struktur- zum Transferkurzarbeitergeld zu einer Verkürzung dieser ebenfalls als Frühverrentungsmaßnahme genutzten Leistung von 24 auf 12 Monate.

Der Bezug von Transferleistungen als Überbrückung bis zum Eintritt in die Rente wird aufgrund der Veränderungen in den Altersrenten, der Verkürzung der Bezugsdauer des Arbeitslosengeldes I und des Wegfalls der einkommensbezogenen Arbeitslosenhilfe weniger attraktiv. Gleichzeitig führt die Anhebung der Altersgrenzen in der Rentenversicherung dazu, dass der Zeitpunkt des Ausscheidens aus dem Arbeitsmarkt nach hinten verschoben wird (Büttner 2005b). Dies zeigt sich bereits ansatzweise in der Statistik des Erwerbsaustrittsalters und der Beschäftigungsquote älterer Erwerbspersonen in Deutschland.

Gleichzeitig manifestiert sich jedoch eine hohe und steigende Transferabhängigkeit älterer Erwerbspersonen unterhalb des gesetzlichen Rentenalters. Hier zeigen sich in den letzten Jahren steigende Bestandsziffern (Tabelle 2). Diese Transferleistungen sorgen dafür, dass derzeit der Übergang aus Vollerwerbstätigkeit in die Regelaltersrente eher selten ist und verschiedene Zwischenstufen des Altersübergangs über andere Transferleistungen genutzt werden können. Der jüngste Anstieg kann zum Teil mit der Beendigung einiger attraktiver Ausstiegsmöglichkeiten erklärt werden, z.B. der steuerlichen Begünstigung von Abfindungen, der längeren Bezugsdauer des Arbeitslosengeldes I oder der zwischenzeitlichen Ankündigung, die Regelung des § 428 SGB III bereits 2005 auslaufen zu lassen.

- Tabelle 2 hier einfügen -

Obwohl einige Transferleistungen an Großzügigkeit verloren haben, bestehen auch in den nächsten Jahren noch Optionen des vorzeitigen Ausscheidens aus dem Arbeitsmarkt. Die Anreize, die verbleibenden Pfade stärker zu nutzen, nehmen damit zu. Dies gilt für die Altersteilzeit, die Erwerbsminderungsrente oder die Rente bei Schwerbehinderung. Erst in den nächsten Jahren dürften sich hier mit weiteren Reformen und der langsamen Anpassung des Beschäftigungssystems Entlastungen ergeben. In dem Maße wie diese Brücken in den Ruhestand abgeschafft werden, sind ältere Arbeitskräfte stärker auf Wieder- oder Weiterbeschäftigung angewiesen. Problematisch sind jedoch die noch eine Dekade umfassen- 
den Übergangsfristen und die weiterhin geringen Anreize zur Arbeit über 62 oder 63 Jahre hinaus (OECD 2005).

\subsection{Aktive Arbeitsmarktpolitik}

Die aktive Arbeitsmarktpolitik kann dazu beitragen, Phasen der Arbeitslosigkeit mit dem Einsatz geeigneter Instrumente zu vermeiden oder zu verkürzen. Bezogen auf ältere Erwerbspersonen ist vor allem von Bedeutung, inwieweit die Instrumente und deren praktische Anwendung dazu beitragen, das Matching zwischen älteren Arbeitskräften und verfügbaren Beschäftigungsmöglichkeiten zu erleichtern, indem Qualifikationsdefizite behoben, mögliche Produktivitätsdefizite oder zu hohe Anspruchslöhne korrigiert und berufliche Mobilität unterstützt werden. Im Instrumentarium des SGB III, welche auf die Bezieher der Versicherungsleistung Arbeitslosengeld I Anwendung findet, lassen sich Maßnahmen für Arbeitslose jeden Alters und Maßnahmen, welche ausschließlich bei Älteren Anwendung finden, unterscheiden.

$\mathrm{Zu}$ den allgemeinen Maßnahmen gehört zum einen die Förderung der beruflichen Fort- und Weiterbildung ( $\S \S 77$ bis 87 SGB III), welche darauf abzielt, über die Verbesserung des Humankapitals und höhere Produktivität die Weiter- oder Wiederbeschäftigungschancen Arbeitsloser oder von Arbeitslosigkeit bedrohter Personen zu erhöhen. Zu diesem Zweck können Arbeitslose Bildungsgutscheine erhalten, sofern im jeweiligen Fall von der Maßnahmenteilnahme eine positive Wirkung auf die Arbeitsmarktintegration erwartet werden kann. Zum anderen zählen hierzu auch Trainingsmaßnahmen und Maßnahmen zur Eignungsfeststellung (§ 48 SGB III), auf zwei bis acht Wochen angelegte Instrumente, welche die Eignung für eine bestimmte Tätigkeit prüfen und notwendige Kenntnisse für eine Arbeitsaufnahme vermitteln sollen.

Lohnkostenzuschüsse sollen über die teilweise Erstattung der Arbeitskosten den Arbeitgebern Anreize zur Einstellung von Arbeitslosen mit Vermittlungshemmnissen bieten. Bei den Eingliederungszuschüssen gelten nach den letzten Neuregelungen 2003 und 2004 derzeit befristet bis Ende 2009 - für die Bezuschussung der Beschäftigung älterer Arbeitsloser großzügigere Bedingungen als für jüngere Arbeitslose (§§ 217 bis 222 und $421 \mathrm{f}$ SGB III). Die Einstellung von Arbeitslosen über 50 Jahren kann seit 2004 über maximal 36 Monate zu höchstens 50\% des Arbeitsentgelts gefördert werden, wobei der Eingliederungszuschuss nach dem ersten Jahr um 10 Prozentpunkte gesenkt wird. 
Zu den für alle Altersgruppen verfügbaren Maßnahmen zählen auch Arbeitsbeschaffungsund Strukturanpassungsmaßnahmen (ABM und SAM) sowie die Förderung der selbstständigen Tätigkeit durch Überbrückungsgeld und Existenzgründungszuschuss („Ich-AG“), wobei letzterer Mitte 2006 wegfallen soll.

Daneben sind im Zuge der Hartz-Reformen Anfang 2003 einige Instrumente in das Repertoire eingeführt worden, die speziell auf Ältere ausgerichtet sind:

1. Die Entgeltsicherung für Ältere nach § 421 j SGB III soll für ältere Arbeitslose über 50 Jahren den Anreiz zur Aufnahme einer geringer entlohnten Tätigkeit stärken. Besteht noch ein Restanspruch von mindestens 180 Tagen auf Arbeitslosengeld I, so wird für diese Zeitdauer ein im Vergleich zur früheren Beschäftigung niedrigeres Arbeitsentgelt um die Hälfte der Differenz zur vormaligen Entlohnung ergänzt. Zusätzlich werden die Rentenbeiträge auf ein Entgeltniveau von 90\% der früheren Beschäftigung aufgestockt. Diese partielle und befristete Bezuschussung des Arbeitsentgelts ist eine Pflichtleistung. Sie sollte zunächst Ende 2005 auslaufen, wurden aber zunächst bis Ende 2007 verlängert.

2. Stellt ein Arbeitgeber einen über 55 Jahre alten Arbeitslosen ein, so wird er gemäß der Regelung über den Beitragsbonus nach § 421 k SGB III dauerhaft von seinem Beitrag zur Arbeitslosenversicherung in Höhe von derzeit 3,25\% befreit. Auch diese Regelung ist zeitlich befristet und gilt mindestens noch bis Ende nächsten Jahres.

3. Die Weiterbildung von Beschäftigten im Alter von über 50 Jahren wird nach $§ 417$ SGB III bis Ende 2006 gefördert, sofern sie in Betrieben mit höchstens 100 Arbeitnehmern tätig sind und die Weiterbildung außerhalb des Betriebes stattfindet. In diesem Fall werden dem Arbeitnehmer die Weiterbildungskosten, dem Arbeitgeber die Zeiten des Arbeitsausfalls bei von Arbeitslosigkeit bedrohten Beschäftigten erstattet.

Im Bereich des SGB II, d.h. für Langzeitarbeitslose ohne Anspruch auf die Versicherungsleistung des Arbeitslosengeldes I, können weitgehend die gleichen Förderinstrumente eingesetzt werden. Hiervon gibt es einige wenige Ausnahmen, z.B. die Ich-AG. Auf der anderen Seite bietet das SGB II gegenüber dem SGB III zwei zusätzliche Instrumente: die Zusatzjobs und das Einstiegsgeld. Die Zusatzjobs nach § 16 Abs. 3 SGB II sind Arbeitsgelegenheiten („Ein-Euro-Jobs“) für Langzeitarbeitslose, die keine andere Arbeit finden. Das Einstiegsgeld nach § 29 SGB II bietet als Ermessensleistung die Möglichkeit, bei Aufnahme einer abhängigen oder selbstständigen Erwerbstätigkeit befristet auf maximal 24 Monate einen Zuschlag zum Arbeitslosengeld II zu gewähren. 
Die Nutzung der arbeitsmarktpolitischen Instrumente für Ältere fällt nach Maßnahmentyp unterschiedlich aus. Tabelle 3 zeigt größere Bestände bei der Förderung durch Eingliederungszuschüsse und bei ABM, SAM und Zusatzjobs; dort liegt auch der Anteil der Älteren an allen Teilnehmern wesentlich höher als bei der Förderung der Weiterbildung. Der Zugang Älterer zur geförderten Weiterbildung ist im Zuge der jüngsten Reformen gesunken, da sich die BA zunehmend auf die Förderung „guter Risiken“ beschränkt (IZA/DIW/infas 2005). Deutlich geringer fällt die Nutzung der neuen Instrumente aus. Insgesamt sind die Bestände Älterer in den Einkommensersatzleistungen der passiven Arbeitsmarktpolitik wesentlich größer als bei den Fördermaßnahmen. In diesem Sinne kann noch immer von einer Subventionierung der Inaktivität gesprochen werden.

- Tabelle 3 hier einfügen -

Eine belastbare Beurteilung arbeitsmarktpolitischer Maßnahmen setzt die Bildung von Kontrollgruppen und angemessene ökonometrische Verfahren voraus. Es liegen zwar kaum Wirkungsstudien mit spezieller Betrachtung der Effekte arbeitsmarktpolitischer Interventionen auf die Beschäftigungschancen Älterer vor (Caliendo/Steiner 2005, KonleSeidl 2005), doch kann sich eine zeitnahe Bewertung der aktiven Arbeitsmarktpolitik für Ältere auf die Anfang 2006 vorgelegten Zwischenberichte zur Evaluation der HartzReformen stützen (Bundesregierung 2006).

Dabei zeigt sich, dass die Entgeltsicherung zwar ein grundsätzlich sinnvolles Instrument ist, das am Problem teilweise zu hoher Anspruchslöhne älterer Arbeitsloser ansetzt und die Aufnahme einer im Vergleich zur früheren Tätigkeit geringer entlohnten Beschäftigung erleichtern kann (Brussig/Knuth/Schweer 2006, ZEW/IAB/IAT 2005). Allerdings wird dieses Instrument in der Praxis nur sehr selten genutzt. Im Jahr 2004 wurde lediglich etwa ein Prozent aller neu begründeten sozialversicherungspflichtigen Beschäftigungsverhältnisse der relevanten Altersgruppe erreicht. Dies lässt sich damit erklären, dass die Entgeltsicherung in den Arbeitsagenturen kaum bekannt ist und nicht strategisch genutzt wird. Auch führt die schwierige administrative Handhabung zur Zurückhaltung in der praktischen Anwendung. Die ökonometrische Evaluation hat bislang, auch aufgrund der sehr geringen Fallzahlen, keinen Einfluss der Entgeltsicherung auf die Beschäftigungschancen der geförderten Gruppe nachweisen können; immerhin gibt eine Mehrheit von rund 65\% der im Rahmen der Evaluation Befragten an, dass dank des teilweisen Ausgleichs der Ver- 
diensteinbußen die Aufnahme einer Stelle erleichtert wurde, ohne dass dies jedoch ausschlaggebend gewesen sei.

Ähnlich liegen die Erfahrungen mit dem Beitragsbonus, der in lediglich drei Prozent aller neuen Beschäftigungsverhältnisse zwischen 55 und 64 Jahren Anwendung fand (7.445 Fälle im Jahr 2003). Auch lassen sich kaum Effekte auf das Einstellungsverhalten der Unternehmen identifizieren. Die geringe Höhe und Sichtbarkeit des Beitragsbonus, welcher über den allgemeinen Beitragseinzug abgewickelt wird, spielen hierbei eine Rolle. Hinzu kommen erhebliche Mitnahmeeffekte.

Im Vergleich hierzu zeigen sich für die Eingliederungszuschüsse bei der Förderung Älterer über 50 Jahren bessere Ergebnisse (ZEW/IAT/IAB 2005). Eingliederungszuschüsse können die Beschäftigungschancen älterer Arbeitsloser verbessern. Dies zeigt eine Analyse, welche sich auf die Neuregelung des Jahres 2002 bezieht, die bei der Einstellung von Älteren bereits nach kürzerer Arbeitslosigkeit Zugang zu Eingliederungszuschüssen eröffnete. Die Ergebnisse sind jedoch nur für Ostdeutschland gesichert. Dabei fiel eine erhebliche Förderung von über $15.000 €$ pro Person im Jahr 2003 an. Auch nach Ablauf der Förderdauer bzw. der Nachbeschäftigungsfrist sind 20 bis 50\% mehr Geförderte sozialversicherungspflichtig beschäftigt als Mitglieder der nicht geförderten Vergleichsgruppe. Es ist jedoch nicht geklärt, ob Eingliederungszuschüsse die Beschäftigung insgesamt erhöht haben oder ob die zusätzliche Beschäftigung zu Lasten nicht geförderter Arbeitsloser ging. Allerdings sind hier erhebliche Mitnahmeeffekte zu erkennen, d.h. es werden auch Einstellungen gefördert, die ohnehin zustande gekommen wären. Zusätzlich weisen die Evaluationsstudien darauf hin, dass die großzügige Förderung der Einstellung Älterer durch Eingliederungszuschüsse dazu beiträgt, dass Arbeitgeber hier von vornherein massive Zuschüsse erwarten, diese gezielt bei der Einstellung Älterer verlangen und nur noch in geringem Umfang bereit sind, Ältere ohne Zuschüsse einzustellen. Dies relativiert die Beschäftigungswirkungen und den möglichen fiskalischen Nutzen der Eingliederungszuschüsse erheblich. Immerhin können sie zur stärkeren Nachfrage der Betriebe nach Älteren beitragen - allerdings auf Kosten der Verdrängung weniger stark geförderter Altersgruppen.

Maßnahmen wie ABM, SAM und Zusatzjobs stellen einen größeren Teil des Teilnehmerbestandes älterer Arbeitsloser dar. Von diesen Maßnahmen kann jedoch auf der Grundlage vorliegender Evaluationsstudien, die durch die jüngsten Studien bestätigt wurden (Bundesregierung 2006), kaum eine Integration in den regulären Arbeitsmarkt erwartet werden. Sie dienen eher dazu, ansonsten in der Statistik erscheinende Arbeitslosigkeit zu verdecken. 
Die Rolle der aktiven Arbeitsmarktpolitik zur Integration Älterer in den Arbeitsmarkt ist durchaus ambivalent. Nach wie vor fehlt eine konsequente Beratungs-, Vermittlungs- und Aktivierungsstrategie der Bundesagentur für Arbeit für teilweise Erwerbsgeminderte und Ältere. Daran haben auch die neuen Instrumente nichts geändert (Brussig/Knuth/Schweer 2006). Nach wie vor hemmen $\S 428$ SGB III, die Altersteilzeit und die implizite Erwartung eines baldigen Renteneintritts eine konsequente Aktivierung älterer Erwerbspersonen. Der Abgang in Alters- oder Erwerbsminderungsrente ist teilweise explizites Ziel der Handlungsprogramme der BA für ältere Arbeitslose, die meist pauschal als „Betreuungskunden“ eingruppiert werden und kaum noch gefördert (und gefordert) werden (Kaltenborn/Knerr/Schiwarov 2006). Dies erscheint zwar aus Sicht der BA plausibel, wirkt jedoch in Bezug auf die Förderung der Beschäftigung Älterer und die Entlastung der sozialen Sicherungssysteme kontraproduktiv. Allerdings haben auch die Träger des SGB II - die Arbeitsgemeinschaften von BA und Kommunen bzw. die Optionskommunen - starke Anreize, ältere Arbeitslose in das Rentensystem zu verschieben anstatt sie mit entsprechendem Betreuungs- und Qualifizierungsaufwand in den Arbeitsmarkt zu integrieren. Dies geht mit der nach wie vor auch in der Arbeitsverwaltung verbreiteten Annahme einher, dass für Ältere ohnehin keine Arbeit mehr gefunden werden kann. Entsprechend begrenzt sind aktivierende und fördernde Interventionen. Die starke Nutzung von Eingliederungszuschüssen ist zwar effektiv, geht jedoch mit hoher Anfälligkeit für Mitnahmeeffekte einher. Humankapitalorientierte Maßnahmen erreichen Ältere kaum noch. Sie setzen ohnehin tendenziell zu spät an, also in der Regel erst nach eingetretener Arbeitslosigkeit oder Erreichen einer bestimmten Altersgrenze. Problematisch ist die Struktur der Arbeitsmarktpolitik auch insofern, als viele Maßnahmen für über 50 oder 55 Jahre alte Arbeitslose signalisieren, dass pauschal eine schwächere Produktivität älterer Arbeitskräfte unterstellt wird. Die Anerkennung eines besonderen Förderbedarfs für Ältere kann zu Vorbehalten der Arbeitgeber gegenüber dieser Gruppe und so zu mittelbarer Diskriminierung beitragen.

\subsection{Humankapital und Produktivität}

Angesichts des beschleunigten Strukturwandels kann nicht mehr davon ausgegangen werden, dass einmal erworbenes berufliches Wissen genügt, um auf Dauer den sich wandelnden Anforderungen auf dem Arbeitsmarkt gerecht werden zu können. Sowohl die Chancen auf längere Beschäftigung im Betrieb als auch die Möglichkeit, einen neuen Arbeitsplatz 
zu finden, hängen von der individuellen „Beschäftigungsfähigkeit“ ab. Eine längere Erwerbstätigkeit im Lebensverlauf lässt auch eine höhere berufliche und intersektorale Mobilität von Erwerbspersonen in höherem Alter notwendig erscheinen. Dies gilt umso mehr als die derzeit älteren Arbeitskräfte noch immer tendenziell schlechter formal qualifiziert sind als jüngere Kohorten und eher in schrumpfenden Branchen beschäftigt sind (OECD 2005). Gehen die Möglichkeiten lebenslanger Beschäftigung im einmal erlernten Tätigkeitsfeld zurück, so besteht bei Weiter- oder Wiederbeschäftigung erheblicher Anpassungsbedarf.

Dem stehen jedoch sehr große Defizite bei „lebenslangem Lernen“ in Deutschland gegenüber, die mit den institutionellen Strukturen des Aus- und Weiterbildungssystems erklärt werden können. Auf der einen Seite erleichtert die hoch entwickelte und differenzierte beruflichen Erstausbildung zwar den Einstieg in das Berufsleben. Sie führt jedoch zu einer starken Festlegung des beruflichen Tätigkeitsspektrums. Das erschwert die Anpassung im weiteren Verlauf des Erwerbslebens, welche zunehmend wichtiger wird. Dieses in Deutschland besonders ausgeprägte Problem wird durch den steilen Abfall der Beteiligung an arbeitsmarktbezogener Weiterbildung mit dem Alter (Abbildung 5) und die Konzentration von Weiterbildung auf höher Qualifizierte noch verschärft. Die einem besonders hohen Arbeitslosigkeitsrisiko ausgesetzte Gruppe der Älteren mit geringer formaler Qualifikation hat in Deutschland faktisch kaum Zugang zu Weiterbildung.

\section{- Abbildung 5 hier einfügen -}

Hierin zeigt sich auch eine Wechselwirkung mit einer lange Zeit auf Frühverrentung ausgerichteten Arbeitsmarktpolitik, da Investitionen in Humankapital im späteren Erwerbsverlauf für Arbeitgeber und Arbeitnehmer umso weniger lohnend sind, je früher ein Ausscheiden aus dem Erwerbsleben erwartet werden kann. Angesichts veränderter Transferleistungssysteme und des demographischen Wandels, die beide auf eine Verlängerung des Erwerbslebens hinauslaufen, werden sich die betriebliche Personalpolitik und das Weiterbildungsverhalten der Beschäftigten selbst anpassen (müssen). Nur so kann einerseits der Personalbedarf der Betriebe angesichts einer rückläufigen Zahl Jüngerer gedeckt und andererseits die Beschäftigungsfähigkeit im verlängerten Erwerbsleben erhalten werden. Die aktive Arbeitsmarktpolitik kann in ihrer derzeitigen Struktur die Defizite in den Betrieben und auf individueller Ebene nicht ausgleichen, da sie zu spät ansetzt und auf Arbeitslose konzentriert ist. Sie könnte jedoch im Zusammenwirken mit den Betrieben, den Beschäf- 
tigten und den Tarifpartnern systematisch eine größere Rolle bei der Humankapitalbildung übernehmen.

Humankapital ist auch ein wichtiger Faktor im Bezug auf die Arbeitsproduktivität. Die empirischen Befunde zur Arbeitsproduktivität Älterer zeigen, dass es keine klaren Belege für eine im Alter sinkende Produktivität gibt (Börsch-Supan/Düzgün/Weiss 2005, Arnds/Bonin 2003b). Dabei ist zwischen individueller Produktivität und der Produktivität in Teams zu unterscheiden. Sowohl die individuelle Produktivität als auch der produktive Beitrag von einzelnen Beschäftigten in Teams setzen sich aus verschiedenen Elementen wie technischen Kenntnissen, Lern- und Anpassungsfähigkeit, Arbeitsdisziplin, Erfahrung und sozialer Kompetenz zusammen. Zwar verändert sich die Bedeutung dieser einzelnen Elemente über das Erwerbsleben hinweg, doch ist kein zwangsläufiger Rückgang der individuellen Leistungsfähigkeit insgesamt nachzuweisen. Das bedeutet, dass Ältere grundsätzlich über das gesamte Erwerbsleben hinweg produktiv sein können, soweit dies durch entsprechende Arbeitsgestaltung und ausreichende Humankapitalinvestitionen unterstützt wird.

Auch Befragungen von Personalverantwortlichen im Rahmen des IAB-Betriebspanels haben gezeigt, dass positive Eigenschaften wie Arbeitsmoral, Arbeitsdisziplin, Qualitätsbewusstsein, Loyalität sowie Erfahrungswissen eher Älteren zugeschrieben werden, während Jüngeren kaum ein Vorsprung bei Flexibilität und Lernfähigkeit zugeschrieben wird; Betriebe mit älteren Belegschaften bewerten ältere Arbeitnehmer positiver. Auch wenn diese Ergebnisse möglicherweise durch sozial erwünschtes Antwortverhalten verzerrt sind, so kann nicht grundsätzlich von einem Defizit Älterer bei individueller Leistungsfähigkeit ausgegangen werden. Das im Vergleich zu Jüngeren veränderte Leistungsportfolio der Älteren kann durch altersgerechte Arbeitsgestaltung und altersgemischte Teams produktiv genutzt werden (Bellmann/Kistler/Wahse 2003). Hier besteht jedoch, auch begünstigt durch die bis dato ausreichende Verfügbarkeit jüngerer Bewerber und die Tendenz zur Frühverrentung, noch immer in vielen Betrieben ein Defizit bei der Anpassung an alternde Belegschaften. Institutionell bedingte Fehlanreize haben die Annahme einer eingeschränkten Leistungsfähigkeit Älterer begünstigt. Die potentiell erreichbare Leistungsfähigkeit über einen langen Erwerbsverlauf hinweg kann nicht mobilisiert werden, wenn aufgrund der Erwartung eines vorzeitigen Ausscheidens aus dem Arbeitsmarkt Investitionen in Weiterbildung unterbleiben. In einem solchen Fall kann die Produktivität tatsächlich zurückgehen und damit entsprechende Annahmen als selbst erfüllende Prophezeiung bestätigen. 
Ältere können folglich als prinzipiell ebenso leistungsfähig wie Jüngere eingeschätzt werden. Dies zeigt sich auch in den Unternehmensbefragungen im Rahmen der HartzEvaluation, bei der über 70\% der Unternehmen angaben, Ältere seien sehr leistungsfähig, wenn sie im Betrieb adäquat eingesetzt werden (RWI/ISG 2005). Ältere sind damit nicht weniger, sondern eher „anders“ leistungsfähig, was in Arbeitszusammenhängen mit altersgemischten Belegschaften von Vorteil sein kann. Soweit sich Rückgänge in der Arbeitsleistung Älterer tatsächlich beobachten lassen, sind diese auf individuelle Gesundheit, Trainiertheit bzw. Erfahrung mit Lernen sowie Teilhabe an Weiterbildung zurückzuführen. Damit kommt der Weiterqualifizierung und der altersgerechten Arbeitsgestaltung eine wichtige Rolle zu. So kann das Leistungsvermögen über einen längeren Verlauf des Erwerbslebens hinweg prinzipiell stabil und durch geeignete Anpassung up to date gehalten oder verbessert werden. Voraussetzung ist, dass die hierzu notwendigen Investitionen nicht zu spät und zu spärlich ausfallen und auch die weniger hoch Qualifizierten erreichen, die ein besonders hohes Risiko der Arbeitslosigkeit im Verlauf des Erwerbslebens aufweisen.

\section{Handlungsbedarf: Altersneutrale Regeln auf dem Arbeits- markt}

Geht man davon aus, dass die Beschäftigungsentscheidung aus ökonomischer Sicht von den genannten institutionellen Einflussgrößen beeinflusst wird, so zeigt die Prüfung für Deutschland, dass kein grundsätzliches Arbeitskostenproblem bei Älteren aufgrund von Senioritätsentlohnung vorzuliegen scheint. Es besteht jedoch ein massives Problem bei der Anpassung und Entwicklung des Humankapitals im Erwerbsverlauf, wodurch Produktivitätsdefizite auftreten können, die eine Weiter- und Wiederbeschäftigung Älterer hemmen. Der Rückgang der Produktivität mit dem Alter ist nicht naturgesetzlich vorgezeichnet, sondern ein Effekt der institutionellen Rahmensetzung. Dies wiederum hängt mit dem noch lange nicht überwundenen Erbe einer Frühverrentungsstrategie zusammen, welche ältere Arbeitskräfte über mehrere Jahrzehnte hinweg zu einem frühen Zeitpunkt aus dem Arbeitsmarkt herausnahm. Die Möglichkeit zum „sozialverträglichen“ Abbau älterer Arbeitskräfte über die Verkettung verschiedener großzügiger Transferleistungen erlaubte zwar die Verjüngung der Belegschaften, senkte jedoch gleichzeitig für Arbeitgeber und Arbeitnehmer die Anreize zur Investition in Weiterbildung. Die in Deutschland nach wie vor geringen Investitionen in das Humankapital im Verlauf des Erwerbslebens werden damit dem Bedarf an Anpassung für Weiter- oder Wiederbeschäftigung nicht gerecht. Die Arbeits- 
marktpolitik besteht noch immer überwiegend darin, Inaktivität zu subventionieren anstatt einen Beitrag zur Stabilisierung der Erwerbstätigkeit Älterer zu leisten. Insbesondere ist die aktive Arbeitsmarktpolitik in ihrer derzeitigen Struktur nicht in der Lage, die massiven Defizite bei der Humankapitalbildung zu kompensieren. Durch die Konzentration der besonderen Förderung auf Ältere über 50 setzt sie nicht nur zu spät an, sondern verstärkt durch die Signalisierung von besonders hohem Förderbedarf bei Älteren möglicherweise in indirekt diskriminierender Weise pauschale Vorbehalte gegen Ältere als „Problemgruppe“ und wirkt so kontraproduktiv. Gleichzeitig zeigt sich das Erbe der Frühverrentung noch immer darin, dass das vorzeitige Ausscheiden faktisch immer noch eine akzeptierte Handlungsoption darstellt. Dies zeigt sich insbesondere in der nach wie vor geringeren Erwartung an die Verfügbarkeit für den Arbeitsmarkt und im Fehlen einer aktiven Vermittlungsstrategie der BA für Ältere. Allerdings zeigt sich bei insgesamt nach wie vor dominanter Förderung von Inaktivität eine allmähliche Veränderung aufgrund der Einschränkung von Frühverrentungsoptionen im Transfersystem.

Internationale Vergleiche zur Beschäftigung Älterer zeigen zweierlei: Zum einen weist die Erfahrung der angelsächsischen Länder USA und Großbritannien, der skandinavischen Staaten Schweden und Dänemark oder auch der Schweiz darauf hin, dass ein höheres Beschäftigungsniveau älterer Personen dauerhaft gehalten werden kann. Zum anderen ist es auch möglich, Phasen der massiven Frühverrentung durch geeignete institutionelle Reformen zu überwinden und die Arbeitsmarktintegration älterer Arbeitskräfte deutlich und nachhaltig zu verbessern, wie etwa die Niederlande, Irland oder Finnland zeigen. Voraussetzung hierfür sind konsistente Reformen und deren konsequente Umsetzung sowie ausreichend Zeit für die Anpassung der Akteure an die veränderten Rahmenbedingungen (Ebbinghaus 2002, Funk 2004, OECD 2005).

Dabei besteht entgegen einer nach wie vor weit verbreiteten Annahme kein Zielkonflikt zwischen einer besseren Beschäftigungssituation der Älteren und guten Arbeitsmarktchancen für jüngere Erwerbspersonen. Der internationale Vergleich weist im Gegenteil auf einen positiven Zusammenhang hin (Abbildung 6). Insofern ist die verbreitete Annahme falsch, ein vorzeitiges Ausscheiden der Älteren würde die Beschäftigung von Jüngeren begünstigen. Beide Gruppen, Jüngere und Ältere, profitieren von größerer Dynamik auf flexibleren Arbeitsmärkten und den dann sinkenden Steuer- und Abgabenlasten.

- Abbildung 6 hier einfügen - 
Unter diesen Bedingungen kann die Beschäftigung älterer Erwerbspersonen auch in Deutschland gesteigert werden. Um das faktische Erwerbsaustrittsalter dem gesetzlichen Rentenalter anzunähern, sind mehrere aufeinander abgestimmte Reformen notwendig. Manche gut gemeinte Privilegierung Älterer hat sich im Hinblick auf die Beschäftigungseffekte ins Gegenteil verkehrt, da bestimmte Besserstellungen bei Transferansprüchen oder im Arbeitsrecht die Erwerbsintegration der Älteren hemmen. In diesem Sinne ist ein einheitliches und „altersneutrales“ Regelwerk für den Arbeitsmarkt erforderlich, das Arbeitsangebot und Arbeitsnachfrage erhöht sowie das Matching beider Seiten verbessert:

1. Die Beschäftigung Älterer kann nur weiter steigen, wenn die noch bestehenden Anreize zum vorgezogenen Ruhestand zügig und konsequent abgebaut werden. Dies betrifft alle noch bestehenden Optionen zum vorzeitigen Ausscheiden auf Kosten der Sozialsysteme wie die subventionierte Altersteilzeit oder den Bezug von Arbeitslosengeld I und II ohne Verfügbarkeit für den Arbeitsmarkt (§ 428 SGB III). Um ein Ausweichen in andere Transfersysteme wie die Erwerbsminderungsrente zu verhindern, sollten teilweise Erwerbsgeminderte keine Renten mehr in voller Höhe erhalten. Weiterhin sind die Regelungen im Rentenrecht auf noch vorhandene Anreize zum vorzeitigen Rentenbezug zu überprüfen, insbesondere im Hinblick auf tatsächlich versicherungsmathematisch korrekte Abschläge.

2. Der Vergleich mit anderen Ländern zeigt, dass das Arbeitsplatzangebot einer Volkswirtschaft keineswegs fix ist. Unter geänderten Rahmenbedingungen können mehr Arbeitsplätze entstehen. Davon profitieren auch ältere Erwerbspersonen. Für sie sind eine Verminderung der gesetzlichen und tarifvertraglichen Arbeitsmarktregulierung durch eine Reform des Kündigungsschutzes, eine generelle Liberalisierung der befristeten Beschäftigung sowie eine flexible und produktivitätsorientierte Entlohnung von besonderer Bedeutung. Eine konsequente Aktivierung und Vermittlung älterer Erwerbspersonen ist ein weiteres Element einer konsistenten und widerspruchsfreien Strategie hin zu altersneutralen Regeln auf dem Arbeitsmarkt - auch bei hoher Arbeitslosigkeit. Die Erwerbsintegration Älterer kann nicht gelingen, wenn an diese Gruppe in der Praxis geringere Anforderungen hinsichtlich Arbeitssuche, Verfügbarkeit und Zumutbarkeit gestellt werden als an Jüngere. Die bisherige Praxis der Bundesagentur für Arbeit ist in diesem Zusammenhang kontraproduktiv.

3. Die Weiter- und Wiederbeschäftigung älterer Arbeitskräfte wird leichter gelingen, wenn eine systematische und längerfristige Pflege und Entwicklung des Humankapitals institutionalisiert wird, um Qualifikationen auf veränderte Anforderungen in den Be- 
trieben und auf dem Arbeitsmarkt rechtzeitig und effektiv anzupassen. Dies setzt die Entwicklung und breite Anwendung geeigneter Organisations- und Finanzierungsmodelle voraus. Dies liegt primär in der Verantwortung von Betrieben, Beschäftigten und Tarifpartnern. Denkbar sind hier Kontenmodelle mit Entgelt- und Arbeitszeitbestandteilen zur (modularisierten) Weiterbildung unter Beteiligung von Arbeitgebern und Arbeitnehmern oder tarifliche Regelungen zur Weiterbildung wie in der BadenWürttembergischen Metallindustrie oder der Chemischen Industrie. Weiterbildung wird jedoch auch zunehmend in der Verantwortung der einzelnen Arbeitnehmer liegen, die sich zum Erhalt ihrer „Beschäftigungsfähigkeit“ nicht allein auf ihre Arbeitgeber oder die Arbeitsmarktpolitik verlassen können. Investitionen in das Humankapital müssen so selbstverständlich werden wie die private Altersvorsorge. Soweit durch stärkere Investitionen in das Humankapital Arbeitslosigkeit vermieden werden kann, ist auch eine staatliche Unterstützung gerechtfertigt. Ein präventiver und investiver Ansatz ist einer Arbeitsmarktpolitik vorzuziehen, die einmal eingetretene Arbeitslosigkeit durch zielgruppenbezogene Förderung zu beheben sucht, aber stets zu spät ansetzt und die Defizite im früheren Erwerbsverlauf nicht ausgleichen kann, möglicherweise sogar mittelbar diskriminierend wirkt.

Ein höheres Beschäftigungsniveau im späteren Verlauf des Erwerbslebens wird um so leichter zu erreichen sein, je stabiler und konsistenter die institutionellen Rahmenbedingungen auf dieses Ziel ausgerichtet werden und je weniger widersprüchliche Signale von Politik und Tarifpartnern ausgehen. Lässt man den Akteuren etwas Zeit für die Anpassung an die veränderte Rahmensetzung, so werden sich auch die erwarteten Effekte auf Weiterund Wiederbeschäftigung Älterer zeigen. In Reaktion auf die bereits eingeleiteten Reformen ist für die nächsten Jahre - allerdings nur bei konsequenter Fortführung - eine „Normalisierung“ der Arbeitsmarktsituation Älterer zu erwarten. Eine längere Beschäftigung im Lebensverlauf wird damit selbstverständlicher. Eine hohe offene Arbeitslosigkeit, wie sie derzeit zu beobachten ist, stellt in diesem Kontext ein Übergangsphänomen, das nicht als Begründung für die Vertagung weiterer Reformen geeignet ist. Je länger widersprüchliche Anreize gesetzt werden, umso langsamer wird sich der notwendige Anpassungsprozess vollziehen. Und eine höhere Transferabhängigkeit der Älteren bedeutet höhere Steuern und Abgaben für noch Beschäftigte und schlechtere Bedingungen für jüngere Kohorten, deren Erwerbstätigkeit jedoch für die Stabilisierung der Rentensysteme zentral ist. Eine bessere Erwerbsintegration der Älteren ist damit auch eine Frage der Gerechtigkeit zwischen den Kohorten. Ein Antidiskriminierungsgesetz und weitere Aufklärungskampagnen über das 
Leistungsvermögen Älterer können diesen Wandel potenziell unterstützen, aber die skizzierten institutionellen Reformen keinesfalls ersetzen. Solange die Signale aus dem Bereich der aktiven Arbeitsmarktpolitik und der Transferleistungen widersprüchlich sind, wird sich das Verhalten von Beschäftigten und Unternehmen kaum verändern.

\section{Literatur}

Arnds, Pascal; Bonin, Holger (2003a): „Institutionelle Faktoren des Rentenzugangs - Ein Überblick aus ökonomischer Perspektive“, in: Matthias Herfurth; Martin Kohli; Klaus F. Zimmermann (Hg.), Arbeit in einer alternden Gesellschaft. Opladen: Leske und Budrich, 65-91.

Arnds, Pascal; Bonin, Holger (2003b): “Gesamtwirtschaftliche Folgen demographischer Alterungsprozesse”, in: Matthias Herfurth; Martin Kohli; Klaus F. Zimmermann (Hg.), Arbeit in einer alternden Gesellschaft. Opladen: Leske und Budrich, 131-177.

Bellmann, Lutz; Kistler, Ernst; Wahse, Jürgen (2003): „Betriebliche Sicht- und Verhaltensweisen gegenüber älteren Arbeitnehmern“. Aus Politik und Zeitgeschichte 53, B 20/2003: 26-34.

Börsch-Supan Axel (2004): Faire Abschläge in der gesetzlichen Rentenversicherung. Mannheim: MEA.

Börsch-Supan, Axel; Düzgun, Ismail; Weiss, Matthias (2005): Altern und Produktivität: Zum Stand der Forschung. Mannheim: MEA.

Brixy, Udo; Christensen, Björn (2002): Wie viel würden Arbeitslose für einen neuen Arbeitsplatz in Kauf nehmen? IAB Kurzbericht 25, 6.12.2002. Nürnberg: IAB.

Brussig, Martin; Knuth, Matthias; Schweer, Oliver (2006): Arbeitsmarktpolitik für ältere Arbeitslose. IAT-Report 2006-02. Gelsenkirchen: IAT.

Büttner, Renate (2005a): Zunehmende Erwerbsbeteiligung von Älteren. IAT Altersübergangs-Report 2005-04. Gelsenkirchen: IAT.

Büttner, Renate (2005b): Höhere Erwerbsbeteiligung in Westdeutschland - Mehr Arbeitslosigkeit und Frühverrentungen in Ostdeutschland. IAT Altersübergangs-Report 2005-05. Gelsenkirchen: IAT.

Bundesregierung (2006): Die Wirksamkeit moderner Dienstleistungen am Arbeitsmarkt: Bericht 2005 der Bundesregierung zur Wirkung der Umsetzung der Vorschläge der Kommission Moderne Dienstleistungen am Arbeitsmarkt. Berlin.

Caliendo, Marco; Steiner, Viktor (2005): „Aktive Arbeitsmarktpolitik in Deutschland: Bestandsaufnahme und Bewertung der mikroökonomischen Evaluationsergebnisse”, Zeitschrift für Arbeitsmarktforschung 38: 396-418.

Duval, Romain (2003): The Retirement Effects of Old-age Pension and Early Retirement Schemes. OECD Economics Department Working Paper 370, Paris: OECD.

Ebbinghaus, Bernhard (2002): Exit from Labor: Reforming Early Retirement and Social Partnership in Europe, Japan, and the USA. Habilitationsschrift. Köln.

Eichhorst, Werner; Sproß, Cornelia (2005): Arbeitsmarktpolitik für Ältere: Die Weichen führen noch nicht in die gewünschte Richtung. IAB Kurzbericht 16, 5.10.2005. Nürnberg: IAB. 
Estevez-Abe, Margarita; Iversen, Torben; Soskice, David (2001): "Social Protection and the Formation of Skills: A Reinterpretation of the Welfare State”, in: Peter A. Hall; David Soskice (Hg.), Varieties of Capitalism: The Institutional Foundations of Comparative Advantage. Oxford: Oxford University Press, 145-183.

Forschungsinstitut zur Zukunft der Arbeit; Deutsches Institut für Wirtschaftsforschung; Institut für angewandte Sozialwissenschaft (2005): Evaluation der Maßnahmen zur Umsetzung der Vorschläge der Hartz-Kommission: Modul 1b: Förderung beruflicher Weiterbildung und Transferleistungen. Bonn: IZA.

Funk, Lothar (2004): Mehr Beschäftigung für Ältere: Lehren aus dem Ausland. Köln: Deutscher Instituts-Verlag.

Gruber, Jonathan; Wise, David W. (2005): Social Security Programs and Retirement Around the World: Fiscal Implications. NBER Working Paper 11290. Cambridge Mass.: NBER.

Gruber, Jonathan; Wise, David W. (1999): "Social Security Programs and Retirement Around the World". Research in Labor Economics 18: 1-40.

Jahn, Elke J. (2005): „Wie wirkt der Kündigungsschutz?“ Zeitschrift für Arbeitsmarktforschung 38: 284-304.

Kaltenborn, Bruno; Knerr, Petra; Schiwarov, Juliana (2006): Agenturen für Arbeit: Systematisierung des Ressourceneinsatzes. Blickpunkt Arbeit und Wirtschaft 4/2006. Berlin.

Konle-Seidl, Regina (2005): Lessons learned: Internationale Evaluierungsergebnisse zu Wirkungen aktiver und aktivierender Arbeitsmarktpolitik. IAB Forschungsbericht 09/2005. Nürnberg: IAB.

OECD (2004): OECD Employment Outlook 2004. Paris: OECD.

OECD (2005): Ageing and Employment Policies: Germany. Paris: OECD.

Rheinisch-Westfälisches Institut für Wirtschaftsforschung; Institut für Sozialforschung und Gesellschaftspolitik (2005): Evaluation der Umsetzung der Vorschläge der HartzKommission - Arbeitspaket 1: Verbesserung der beschäftigungspolitischen Rahmenbedingungen und Makrowirkungen der aktiven Arbeitsmarktpolitik. Bericht 2005. Essen: RWI.

Zentrum für Europäische Wirtschaftsforschung; Institut für Arbeitsmarkt und Berufsforschung; Institut Arbeit und Technik (2005): Evaluation der Maßnahmen zur Umsetzung der Vorschläge der Hartz-Kommission - Arbeitspaket 1: Wirksamkeit der Instrumente, Modul 1d: Eingliederungszuschuss und Entgeltsicherung. Bericht 2005. Mannheim: ZEW. 


\section{Anhang}

Abbildung 1: Arbeitsmarktintegration nach Altersgruppen in \%, 2004

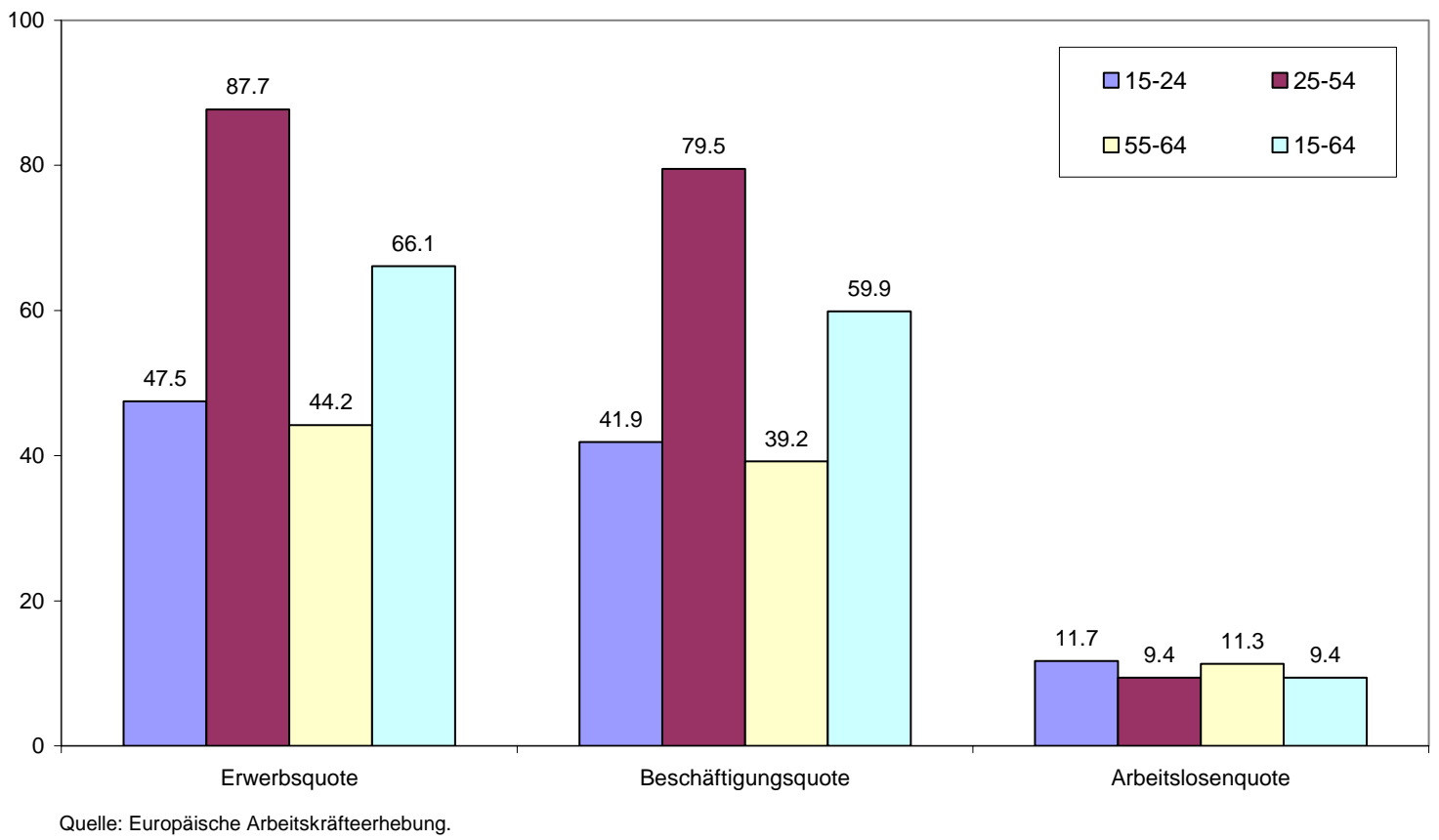

Abbildung 2: Beschäftigungsquoten der 55- bis 64-Jährigen in \%, 1998-2004

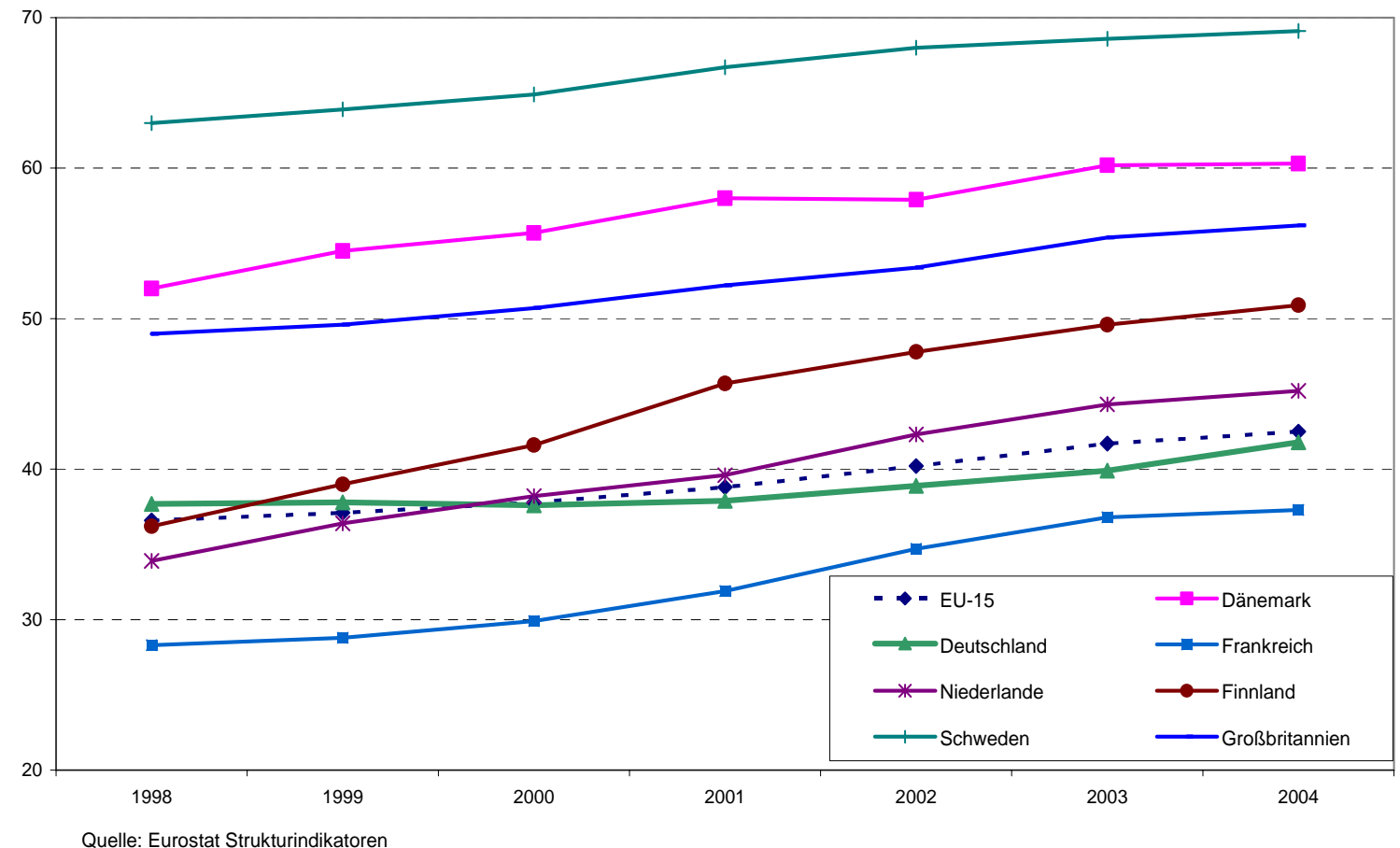


Abbildung 3: Anteil der Langzeitarbeitslosen an allen Arbeitslosen in \%, 2003

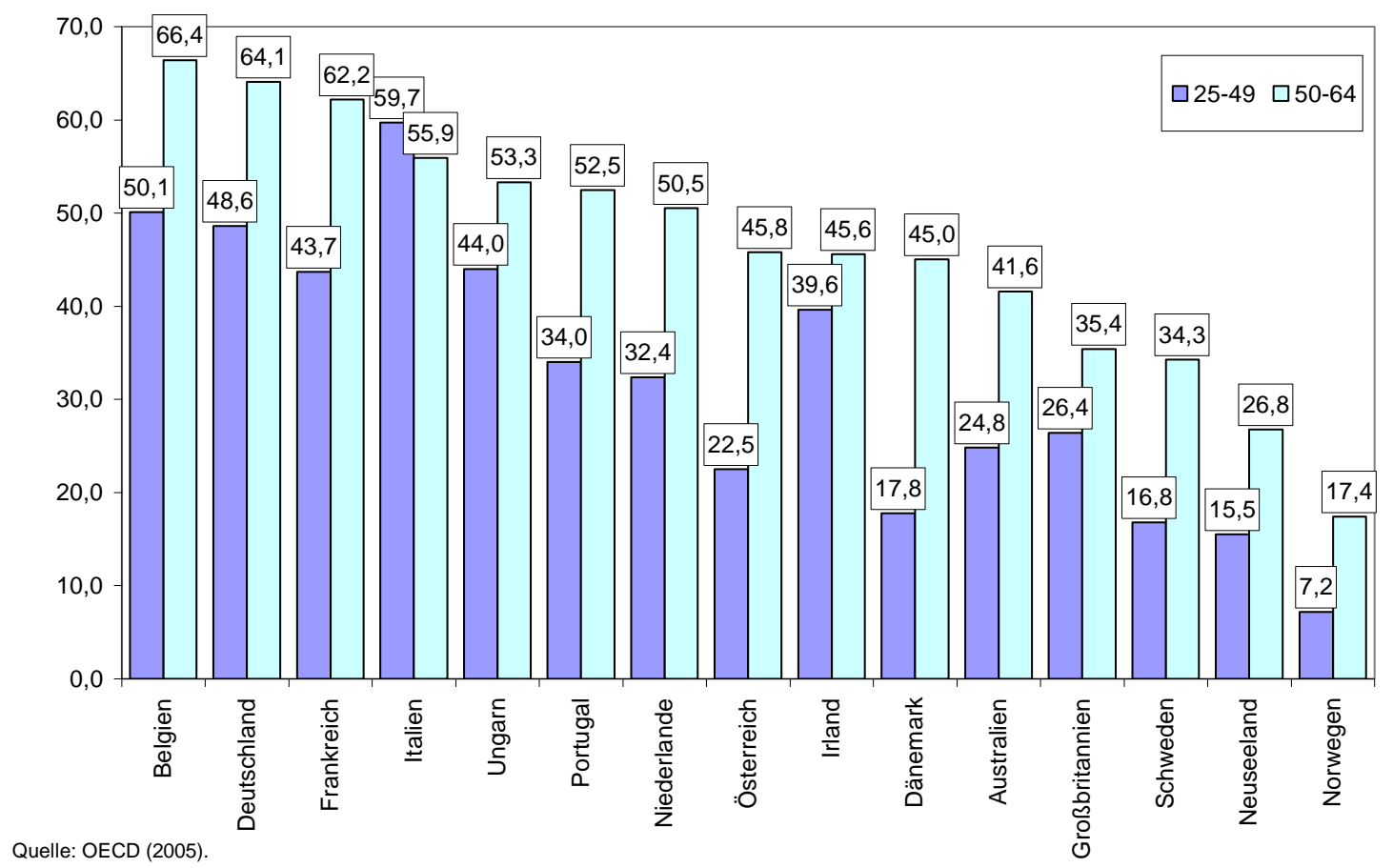

\section{Abbildung 4: Erwerbsaustrittsalter, 2001 und 2004}

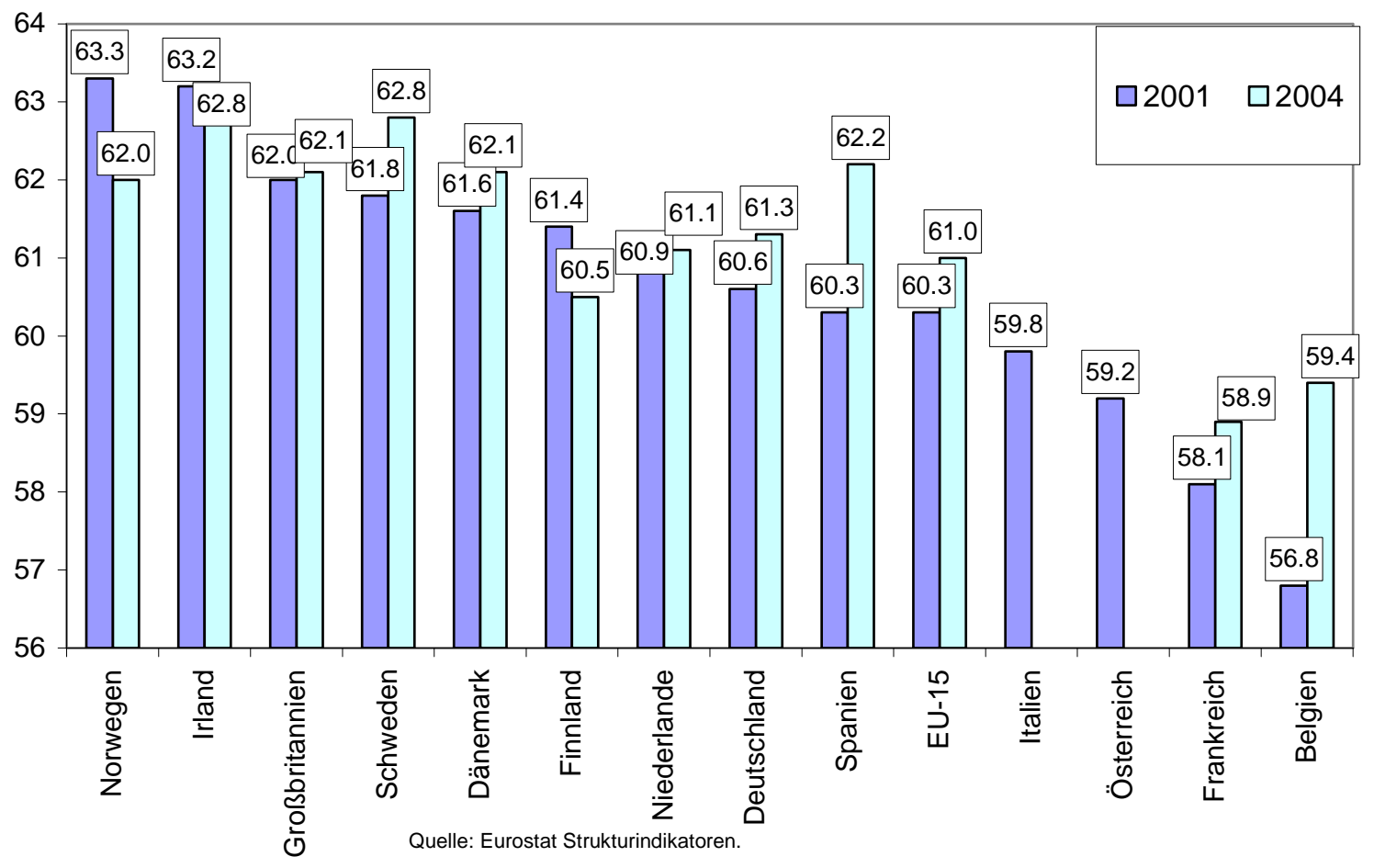


Abbildung 5: Beteiligung an Weiterbildung im Referenzzeitraum von 4 Wochen nach Lebensalter in \%, 2004

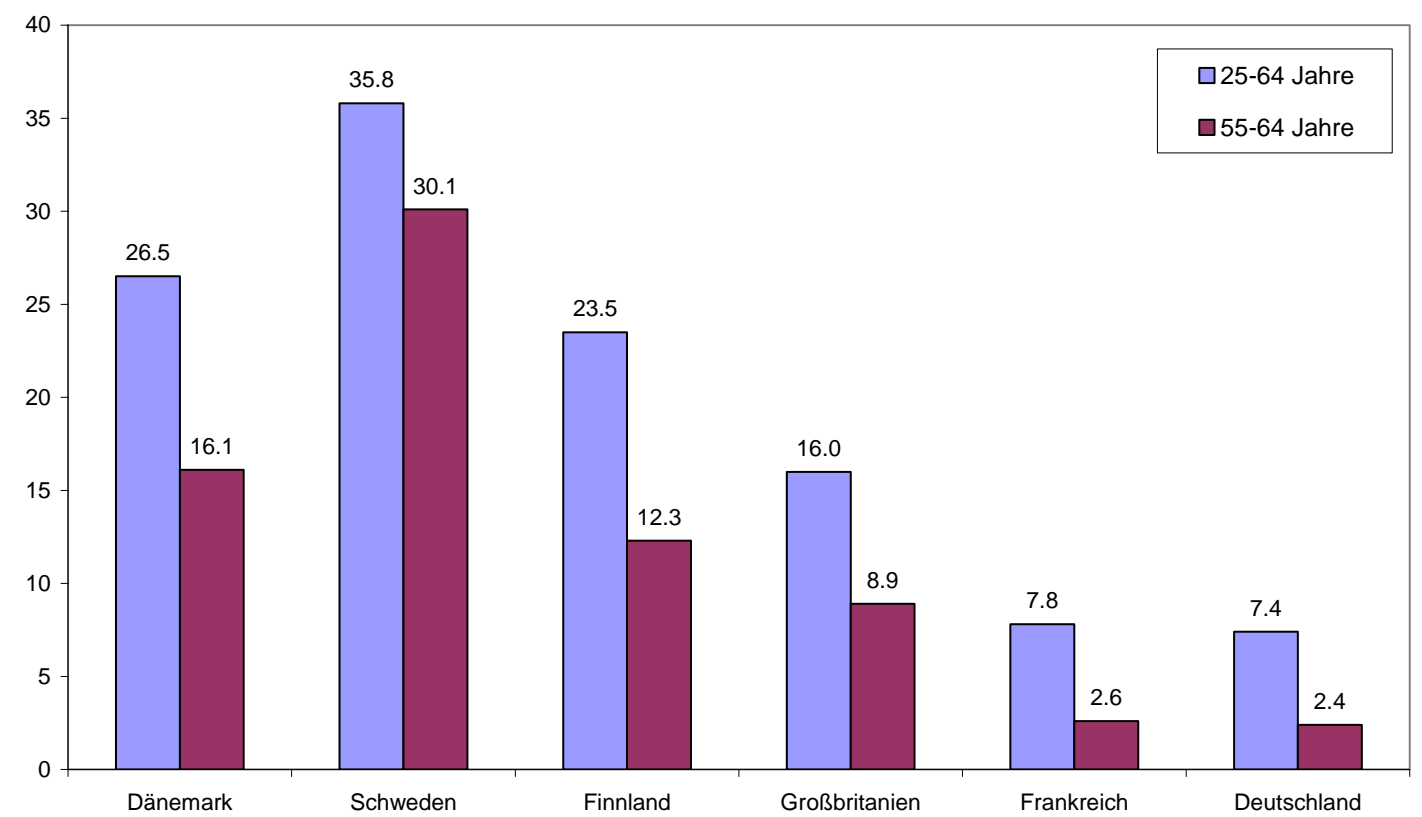

Anmerkung: Angaben umfassen sämtliche Aus- und Erstausbildung, (ständige) Weiterbildung, betriebliche Ausbildung, Lehre, Ausbildung am Arbeitsplatz, Seminare, Fernunterricht, Abendschule usw. sowie allgemein bildende Kurse.

Abbildung 6: Beschäftigungsquoten Jüngerer und Älterer in \%, 2004

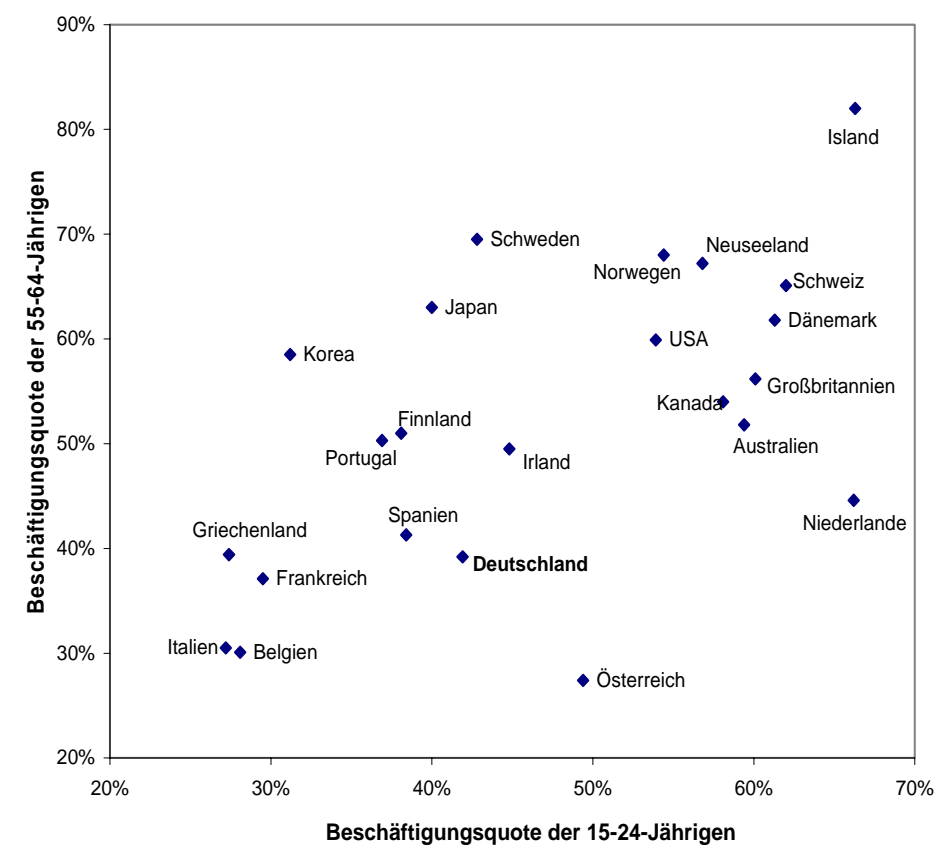

Quelle: OECD Employment Outlook 2005. 
Tabelle 1: Erwerbsquoten nach Geschlecht und Bildungsniveau in \%, 50-64 Jahre, 2002

\begin{tabular}{|l|c|c|c|c|}
\hline Länder & Bildungsniveau & Männer & Frauen & Insgesamt \\
\hline \multirow{3}{*}{ Deutschland } & Niedrig & 55.4 & 35.9 & 42.2 \\
\cline { 2 - 5 } & Mittel & 63.9 & 50.5 & 57.2 \\
\cline { 2 - 5 } Frankreich & Hoch & 77.8 & 67.7 & 74.5 \\
\hline \multirow{3}{*}{ Niederlande } & Niedrig & 55.7 & 44.3 & 49.3 \\
\cline { 2 - 5 } & Mittel & 67.5 & 57.5 & 63.0 \\
\cline { 2 - 5 } & Hoch & 81.6 & 68.9 & 75.5 \\
\hline \multirow{3}{*}{ Schweden } & Niedrig & 60.5 & 32.0 & 43.5 \\
\cline { 2 - 5 } & Mittel & 71.1 & 51.9 & 62.8 \\
\hline \multirow{3}{*}{ Großbritannien } & Hoch & 81.4 & 65.9 & 75.6 \\
\cline { 2 - 5 } & Niedrig & 73.2 & 60.3 & 67.3 \\
\cline { 2 - 5 } & Mittel & 80.0 & 76.8 & 78.4 \\
\hline \multirow{3}{*}{ USA } & Hoch & 88.1 & 87.0 & 87.5 \\
\cline { 2 - 5 } & Niedrig & 57.4 & 49.6 & 53.5 \\
\cline { 2 - 5 } & Mittel & 76.0 & 72.6 & 74.7 \\
\hline \multirow{3}{*}{ OECD } & Hoch & 79.6 & 80.8 & 80.1 \\
\cline { 2 - 5 } & Niedrig & 59.3 & 38.8 & 48.6 \\
\cline { 2 - 5 } & Mittel & 73.8 & 62.5 & 67.6 \\
\hline & Hoch & 84.3 & 74.6 & 79.7 \\
\cline { 2 - 5 } & Niedrig & 63.8 & 38.0 & 50.0 \\
\cline { 2 - 5 } & Mittel & 71.0 & 57.4 & 64.3 \\
\hline
\end{tabular}

Anmerkung: niedriges Bildungsniveau: ohne weiterführenden Schul- oder Berufsabschluss; mittleres Bildungsniveau: weiterführender Schulabschluss oder Berufsausbildung; hohes Bildungsniveau: akademische Ausbildung; Quelle: OECD 2005. 
Tabelle 2: Transferbezug Älterer, jahresdurchschnittliche Bestände, 1998 bis 2005

\begin{tabular}{|l|c|c|c|c|c|c|c|c|}
\hline In 1.000 & $\mathbf{1 9 9 8}$ & $\mathbf{1 9 9 9}$ & $\mathbf{2 0 0 0}$ & $\mathbf{2 0 0 1}$ & $\mathbf{2 0 0 2}$ & $\mathbf{2 0 0 3}$ & $\mathbf{2 0 0 4}$ & $\mathbf{2 0 0 5}$ \\
\hline $\begin{array}{l}\text { Rentenbezug vor dem 65. } \\
\text { Lebensjahr } \\
\text { davon: }\end{array}$ & $\ldots$ & $\ldots$ & 2.554 & 2.510 & 2.397 & 2.261 & 2.054 & $\ldots$ \\
\hline - wegen Arbeitslosigkeit & $\ldots$ & $\ldots$ & 864 & 782 & 672 & 565 & 452 & $\ldots$ \\
\hline - nach Altersteilzeit & $\ldots$ & $\ldots$ & 20 & 58 & 98 & 129 & 141 & $\ldots$ \\
\hline $\begin{array}{l}\text {-wegen verminderter } \\
\text { Erwerbsfähigkeit }\end{array}$ & $\ldots$ & $\ldots$ & 371 & 355 & 343 & 323 & 456 & $\ldots$ \\
\hline $\begin{array}{l}\text { Bezieher von Arbeitslo- } \\
\text { sengeld I (ab 50 Jahren) }\end{array}$ & 823 & 760 & 664 & 622 & 615 & 653 & 656 & 645 \\
\hline $\begin{array}{l}\text { Darunter: erleichterter } \\
\text { Leistungsbezug nach } \delta \\
\text { 428 SGB III }\end{array}$ & 194 & 200 & 192 & 224 & 291 & 370 & 377 & $\ldots$ \\
\hline $\begin{array}{l}\text { Bezieher von Arbeitslo- } \\
\text { senhilfe (ab 50 Jahren) }\end{array}$ & 409 & 431 & 432 & 440 & 495 & 565 & 619 & $\ldots$ \\
\hline $\begin{array}{l}\text { Erwerbsfähige Hilfebe- } \\
\text { zieher (Arbeitslosengeld } \\
\text { II, ab 50 Jahren) }\end{array}$ & $\ldots$ & $\ldots$ & $\ldots$ & $\ldots$ & $\ldots$ & $\ldots$ & $\ldots$ & 1.002 \\
\hline \begin{tabular}{l} 
Geförderte Altersteilzeit \\
\hline
\end{tabular} & 8 & 19 & 33 & 50 & 61 & 69 & 79 & $\ldots$ \\
\hline
\end{tabular}

Anmerkungen und Quellen: Renten: Deutsche Rentenversicherung; Arbeitslosengeld I, II, Arbeitslosenhilfe und Altersteilzeit: Bundesagentur für Arbeit. Angaben für 2005 bei Arbeitslosengeld I nur bis 10/2005, Altersteilzeit und Arbeitslosengeld II bis 9/2005. 
Tabelle 3: Teilnehmerbestände Älterer über 50 Jahren in ausgewählten Maßnahmen der aktiven Arbeitsmarktpolitik, 1998 bis 2005

\begin{tabular}{|c|c|c|c|c|c|c|c|c|c|}
\hline & 1998 & 1999 & 2000 & 2001 & 2002 & 2003 & 2004 & 2005 & \begin{tabular}{|c|} 
Anteil \\
Älterer \\
in \%
\end{tabular} \\
\hline $\begin{array}{l}\text { Entgeltsicherung nach } \\
\text { § 421j SGB III } \\
\end{array}$ & $\ldots$ & $\cdots$ & $\cdots$ & $\ldots$ & $\cdots$ & 4.596 & 6.433 & 4.334 & $\ldots$ \\
\hline \begin{tabular}{|l|} 
Eingliederungs- \\
zuschüsse
\end{tabular} & 39.000 & 31.671 & 35.512 & 47.341 & 71.322 & 77.050 & 57.509 & 14.620 & 34,0 \\
\hline $\begin{array}{l}\text { Eignungsfeststellungs- } \\
\text { und Trainingsmaß- } \\
\text { nahmen }\end{array}$ & $\cdots$ & $\cdots$ & 6.473 & 8.155 & 9.508 & 10.578 & 10.030 & 7.105 & 10,9 \\
\hline $\begin{array}{l}\text { Berufliche Weiterbil- } \\
\text { dung }\end{array}$ & 26.049 & 26.218 & 26.529 & 27.738 & 24.359 & 13.687 & 7.225 & 5.617 & 4,5 \\
\hline $\begin{array}{l}\text { Förderung der Wei- } \\
\text { terbildung nach § } 417 \\
\text { SGB III }\end{array}$ & $\ldots$ & $\ldots$ & $\ldots$ & $\ldots$ & $\ldots$ & 50 & 73 & 83 & $\ldots$ \\
\hline $\begin{array}{l}\text { Arbeitsbeschaffungs- } \\
\text { maßnahmen (ABM) }\end{array}$ & 62.429 & 69.098 & 68.205 & 61.043 & 51.190 & 36.493 & 28.380 & 15.457 & 36,0 \\
\hline $\begin{array}{l}\text { Strukturanpassungs- } \\
\text { maßnahmen (SAM) }\end{array}$ & 31.755 & 33.439 & 26.915 & 24.359 & 26.512 & 25.055 & 19.598 & 5.938 & 73,8 \\
\hline Überbrückungsgeld & $\cdots$ & $\cdots$ & 4.556 & 4.842 & 5.889 & 8.021 & 10.301 & 12.745 & 15,2 \\
\hline $\begin{array}{l}\text { Existenzgründungs- } \\
\text { zuschuss (Ich-AG) }\end{array}$ & $\ldots$ & $\ldots$ & $\ldots$ & $\ldots$ & $\ldots$ & 5.719 & 20.778 & 34.967 & 17,7 \\
\hline $\begin{array}{l}\text { Zusatzjobs („Ein- } \\
\text { Euro-Jobs“) }\end{array}$ & $\cdots$ & $\ldots$ & $\cdots$ & $\cdots$ & $\cdots$ & $\ldots$ & $\cdots$ & 58.832 & 20,3 \\
\hline
\end{tabular}

Anmerkung: Angaben für 2005 auf Basis Dezember 2005, Anteil Älterer im September 2005; Quelle: Bundesagentur für Arbeit. 